ANDRÉ GNECCO AVELAR

ANÁLISE DE COMPONENTES PRINCIPAIS NA DINÂMICA DA VOLATILIDADE IMPLÍCITA E SUA CORRELAÇÃO COM O ATIVO OBJETO 

ANDRÉ GNECCO AVELAR

\title{
ANÁLISE DE COMPONENTES PRINCIPAIS NA DINÂMICA DA VOLATILIDADE IMPLÍCITA E SUA CORRELAÇÃO COM O ATIVO OBJETO
}

\author{
Dissertação apresentada à Escola \\ Politécnica da Universidade de São \\ Paulo para obtenção do título de \\ Mestre em Engenharia \\ Área de Concentração: Engenharia de \\ Sistemas \\ Orientador: Prof. Dr. Oswaldo Luiz do \\ Valle Costa
}

\section{São Paulo}


Este exemplar foi revisado e alterado em relação à versão original, sob responsabilidade única do autor e com a anuência de seu orientador.

São Paulo, de julho de 2009.

Assinatura do autor

Assinatura do orientador

FICHA CATALOGRÁFICA

Avelar, André Gnecco

Análise de componentes principais na dinâmica da volatilidade implicita e sua correlaçāo com o ativo objeto / A.G. Avelar. -ed.rev. -- São Paulo, 2009.

55 p.

Dissertação (Me strado) - Escola Politécnica da Universidade de São Paulo. Departamento de Engenharia de Telecomunicaçöes e Controle.

1. Engenharia financeira 2.Mode los matemáticos 3.Finanças I.Universidade de São Paulo. Escola Politécnica. Departamento de Engenharia de Telecomunicaçōes e Controle Il.t. 


\section{Dedicatória}




\section{Resumo}

Como a volatilidade é a única variável não observada nas fórmulas padrão de apreçamento de opções, o mercado financeiro utiliza amplamente o conceito de volatilidade implícita, isto é, a volatilidade que ao ser aplicada na fórmula de apreçamento resulte no preço correto (observado) das opções negociadas.

Por isso, entender como as volatilidades implícitas das diversas opções de dólar negociadas na BM\&F, o objeto de nosso estudo, variam ao longo do tempo e como estas se relacionam é importante para a análise de risco de carteiras de opções de dólar/real bem como para o apreçamento de derivativos cambiais exóticos ou pouco líquidos.

A proposta de nosso estudo é, portanto, verificar se as observações da literatura técnica em diversos mercados também são válidas para as opções de dólar negociadas na BM\&F: que as volatilidades implícitas não são constantes e que há uma relação entre as variações das volatilidades implícitas e as variações do valor do ativo objeto.

Para alcançar este objetivo, aplicaremos a análise de componentes principais em nosso estudo. Com esta metodologia, reduziremos as variáveis aleatórias que representam o processo das volatilidades implícitas em um número menor de variáveis ortogonais, facilitando a análise dos dados obtidos.

Palavras chave: Engenharia financeira. Superfície de volatilidade implícita. Análise de componentes principais. 


\section{Abstract}

Volatility is the only unobserved variable in the standard option pricing formulas and hence implied volatility is a concept widely adopted by the financial market, meaning the volatility which would make the formula yield the option's real market price.

Therefore, understanding how the implied volatility of the options on dollar traded at $\mathrm{BM} \& \mathrm{~F}$, the subject of our study, vary over time is important for risk analysis over dollar option books and for pricing of exotic or illiquid derivatives

Our work's proposal is to verify if the observations made by the technical literature over several markets could also be applied to the options on dollar traded at BM\&F: implied volatilities do vary over time and there is a relation between this variation and the variation of the underlying asset price.

In order to fulfill these goals, we will apply principal component analysis in our study. This methodology will help us analyze the data by reducing the number of variables that represent the implied volatility process into a few orthogonal variables.

Keywords: Financial engineering. Implied volatility surface. Principal Component Analysis. 


\section{Sumário}

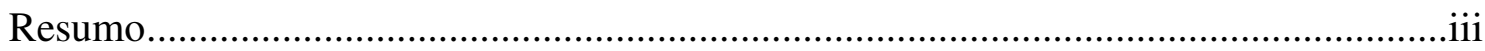

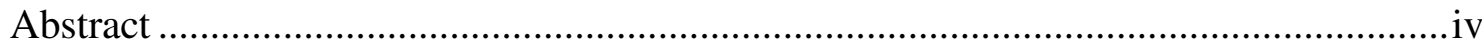

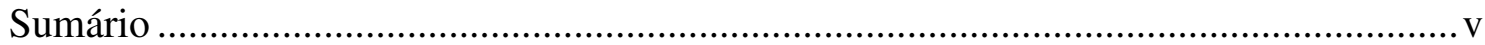

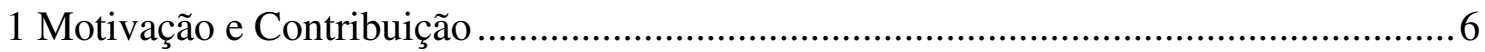

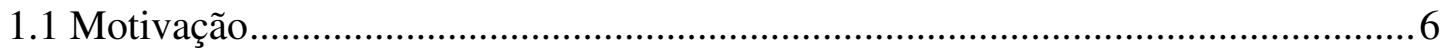

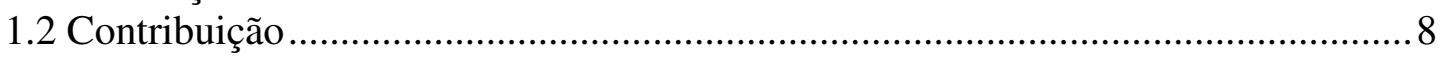

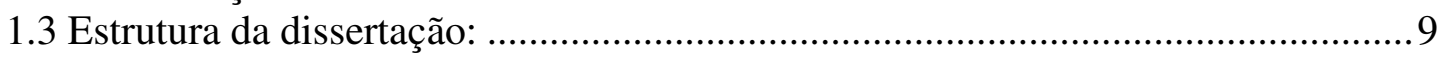

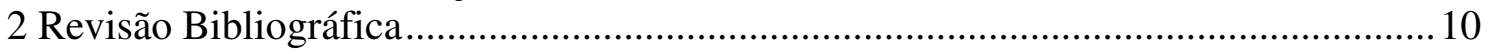

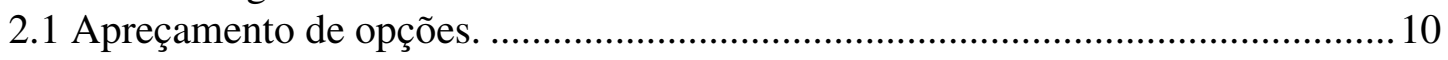

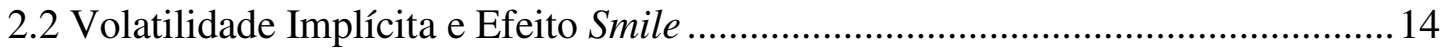

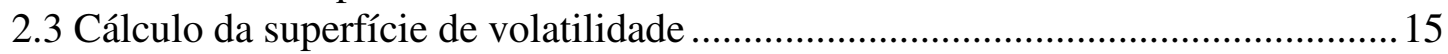

2.4 Correlação entre a volatilidade implícita e o preço do ativo objeto....................... 18

2.5 Comportamento da Superfície de Volatilidade ao longo do tempo .......................20

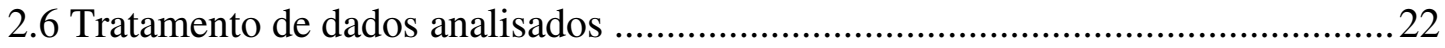

3 Análise de Componentes Principais ................................................................................24

3.1 Metodologia da Análise de Componentes Principais..........................................2 24

3.2 Correlação entre as componentes principais e uma variável aleatória uni-

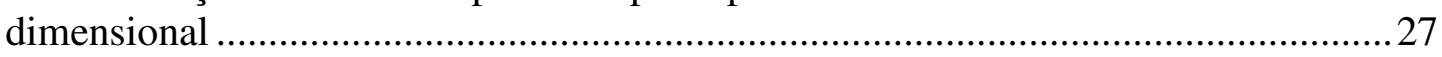

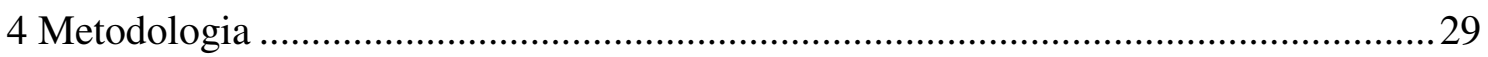

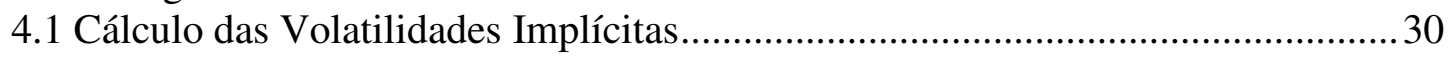

4.2 Interpolação da Superfície de Volatilidade ............................................................. 31

4.3 Decomposição da variação diária da superfície ................................................... 33

4.4 Análise da correlação entre as variações da superfície de volatilidade e as

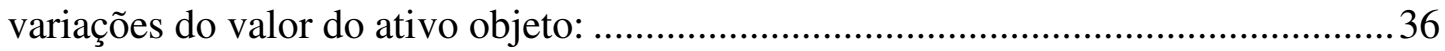

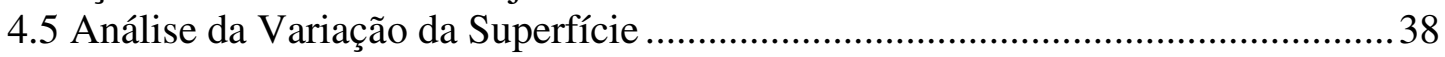

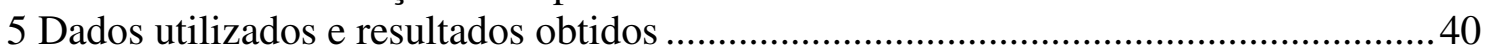

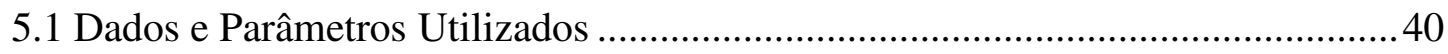

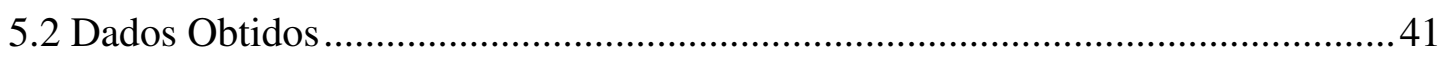

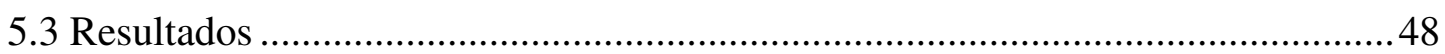

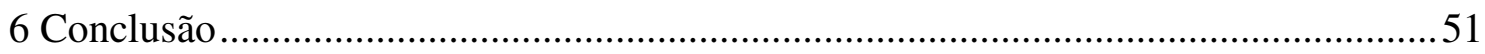

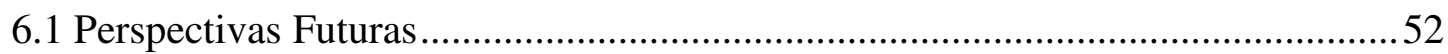

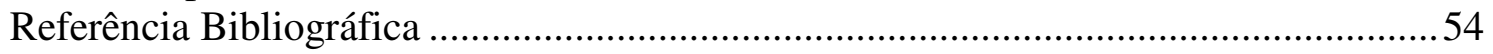




\section{Capítulo 1}

\section{Motivação e Contribuição}

\subsection{Motivação}

Ao calcular-se a volatilidade implícita de todos os contratos de opções sobre um mesmo ativo objeto negociados em um dia, vê-se claramente que há vários níveis de volatilidade implícita. Destaca-se principalmente o efeito "smile" em que a volatilidade implícita é maior para as opções muito "in-the-money" (que dariam retorno positivo ao seu detentor se liquidadas) e as muito "out-of-the-money" (que não dariam retorno), fenômeno amplamente documentado na literatura (DUPIRE, 1993; DERMAN; KANI, 1994).

Se o modelo e dados observados fossem perfeitos, a volatilidade implícita seria única para qualquer opção negociada sobre o mesmo ativo objeto. Como isto não ocorre, conclui-se que há erros de observação - como diferença de tempo entre as negociações e também falhas no modelo de Black-Scholes e seus derivados como, por exemplo, não considerar a possibilidade de saltos nos preços dos ativos ou a falta de liquidez do mercado de opções.

Porém, graças à simplicidade e ampla adoção de tais modelos, as volatilidades implícitas são estudadas por permitirem o controle de risco e posicionamento em carteiras de opções bem como apreçar opções sem liquidez, sejam vanillas ou exóticas, em que a volatilidade implícita é a variável de entrada para os diversos modelos utilizados pelo mercado. 
A volatilidade implícita é de uso tão comum no mercado financeiro que os operadores têm como prática calcular e negociar suas posições em termos de volatilidades e não em preço das opções. Assim, uma compra de opção de 4 meses sobre determinado ativo com strike de $\mathrm{R} \$ 50$ é representada como a compra de uma opção de 4 meses à $30 \%$ de volatilidade, explicitando deste modo que caso a incerteza sobre o preço deste ativo suba (consequentemente sua volatilidade implícita também), o operador terá resultado positivo na operação. É importante deixar claro aqui que um aumento da incerteza sobre o ativo objeto não traz aumento imediato de sua volatilidade histórica, mas certamente de sua volatilidade implícita.

Justifica-se este padrão em apreçar opções por sua volatilidade implícita pelo fato de que em um portfólio contendo opções sobre o mesmo ativo objeto e o próprio ativo, a volatilidade implícita é o segundo fator de risco mais importante, sendo o primeiro a variação no preço do ativo, contra a qual o investidor pode facilmente se proteger. Como este fator de risco é diferente para cada contrato de opção negociado, torna-se importante entender como a volatilidade implícita de cada contrato varia com o tempo e qual a relação entre estas variações.

Assim, o estudo da variação das volatilidades implícitas no tempo é de grande utilidade prática, permitindo controlar o risco e retorno de carteiras de opções (sobre o mesmo ativo objeto) com diversos strikes e maturidades bem como apreçar opções exóticas ou ilíquidas. Para alcançar este objetivo, uma das ferramentas mais comuns é a superfície de volatilidade, na qual mostra-se a volatilidade implícita em todos os pontos do espaço \{Strike/Preço x Maturidade\} e não apenas dos pontos onde há contratos negociados. Com o estudo das variações diárias da superfície de volatilidade, cumpriremos esta primeira parte do objetivo.

Nosso segundo objetivo, avaliar como as variações de preço do ativo objeto (em nosso caso o dólar) se relacionam com as variações da volatilidade implícita também apresentam como interesse abordar aspectos de risco e retorno em carteiras que contém o ativo e opções sobre este ativo bem como avaliar se a perspectiva de variação do ativo objeto pode refletir em alguma perspectiva de variação da volatilidade implícita. 
Como há diversas volatilidades implícitas para cada dia de negócio, estaremos tratando com uma variável multidimensional. De modo a facilitar nosso estudo, utilizaremos a análise de componentes principais. Com esta metodologia aplica-se uma transformação linear reduzindo a quantidade das variáveis analisadas em variáveis ortogonais que mantém o máximo de variância do processo original.

A aplicação prática da análise de componentes principais também é uma motivação em nosso trabalho. Trata-se de uma metodologia que em engenharia financeira é aplicada não só no estudo de volatilidades implícitas mas também na estrutura a termo de taxa de juros e na análise de fatores de risco de carteiras. Esta metodologia também se destaca por sua aplicação em reconhecimento de imagens.

\subsection{Contribuição}

A aplicação de nosso estudo será sobre as opções de dólar negociadas na BM\&F. Os estudos de volatilidade implícita desenvolvidos anteriormente para este mercado tinham foco diferente do nosso, pois apenas analisaram a possibilidade de mudança do regime de câmbio fixo da década de 90, como, por exemplo, o trabalho de Guimarães e Silva (2002).

As análises que faremos da variação da volatilidade implícita e da sua relação com a variação do valor do ativo objeto não foram realizadas em trabalhos anteriores nas opções de câmbio dólar/real, apenas em outros mercados, como índices de ações e outras moedas. Com nosso estudo, portanto, estaremos contribuindo com as conclusões que a literatura extraiu sobre o comportamento da volatilidade implícita, agregando com a análise de mais um mercado àqueles já abordados em outros estudos. 


\subsection{Estrutura da dissertação:}

Esta dissertação está organizada do seguinte modo: No Capítulo 2 fazemos a revisão bibliográfica dos temas pertinentes ao nosso estudo: apreçamento de opções e volatilidade implícita, superfície de volatilidade, correlação entre as volatilidades implícitas e movimentações do ativo objeto e comportamento da superfície de volatilidade.

O Capítulo 3 trata da técnica de Análise de Componentes Principais, que aplicaremos em nosso trabalho de modo a transformar os dados originais, permitindo assim selecionar as informações mais adequadas para a análise estatística.

Todas as etapas da metodologia que utilizaremos são expostas no Capítulo 4. Destas etapas, destacam-se a representação da volatilidade implícita via uma superfície e a aplicação prática da Análise de Componentes Principais.

No Capítulo 5, detalham-se os dados e parâmetros utilizados bem como mostramos e descrevemos os resultados obtidos com a aplicação da metodologia. As conclusões acerca destes resultados são expostas no Capítulo 6. 


\section{Capítulo 2}

\section{Revisão Bibliográfica}

Neste capítulo pretendemos analisar como o problema que iremos abordar, o estudo das superfícies de volatilidade e como sua variação no tempo está relacionada com o ativo objeto, foi abordado em trabalhos anteriores. Inicialmente, porém, faremos uma revisão das metodologias necessárias para este tipo de estudo: apreçamento de opções, o conceito de volatilidade implícita e efeito smile, interpolação da superfície de volatilidade e análise de suas componentes principais.

\subsection{Apreçamento de opções.}

Uma opção de compra européia é um tipo de contrato derivativo que dá ao seu comprador, em uma data futura fixa $(\mathrm{T})$, o poder de decidir pela compra ou não de um ativo específico (o ativo objeto) a um preço pré-determinado, chamado de strike $(\mathrm{K})$.

Assim, sendo $S_{t}$ o preço observado do ativo objeto em $t$, temos no instante $T$ :

- $\quad$ Se $S_{T}<K$, o detentor da opção não a exerce, não recebendo nada.

- Se $\mathrm{S}_{\mathrm{T}}>\mathrm{K}$, o dono da opção a exerce e compra por $\mathrm{K}$ um ativo que vale $\mathrm{S}_{\mathrm{T}}$, recebendo $\mathrm{S}_{\mathrm{T}}-\mathrm{K}$.

- Se $S_{\mathrm{T}}=\mathrm{K}$, exercer ou não a opção é indiferente, em ambos os casos, não receberá nada.

Para uma opção européia de venda, o raciocínio é o mesmo, mas o dono da opção pode decidir por vender ou não o ativo no instante T pelo preço K. Seu racional em T seria: 
- Se $\mathrm{S}_{\mathrm{T}}<\mathrm{K}$, o detentor da opção a exerce e vende por $\mathrm{K}$ um ativo que vale $\mathrm{S}_{\mathrm{T}}$, recebendo $\mathrm{K}-\mathrm{S}_{\mathrm{T}}$.

- $\quad$ Se $\mathrm{S}_{\mathrm{T}}>\mathrm{K}$, o dono da opção não a exerce e não recebe nada.

- Se $S_{T}=K$, exercer ou não a opção é indiferente, em ambos os casos, não receberá nada.

Assim, determina-se facilmente qual o valor da opção no seu instante de vencimento. Mas para determinar qual o preço de uma opção européia antes de seu vencimento, diversos métodos para apreçamento de opções foram desenvolvidos nas últimas décadas, sendo que uma das fórmulas mais difundidas é a de Black-Scholes (1973). Seu modelo assume que o preço do ativo objeto segue um movimento browniano geométrico:

$$
d S_{t}=\mu \cdot S_{t} \cdot d t+\sigma \cdot S_{t} \cdot d W_{t}
$$

Onde:

$\mathrm{S}_{\mathrm{t}}=$ Preço do ativo no instante $\mathrm{t}$

$\mu=$ tendência do ativo

$\sigma=$ volatilidade do ativo

$\mathrm{W}_{\mathrm{t}}=$ processo de Wiener

Assume-se também que estamos num mercado perfeito, onde não há oportunidade de arbitragens, é possível operar continuamente, sem custos de transação ou impostos, é possível ficar vendido no ativo objeto, o ativo é perfeitamente divisível e não paga dividendos nem juros e há uma taxa de juros (r) constante e única. Dada estas premissas, o preço de uma opção de compra européia segundo a fórmula de BlackScholes é:

$$
\mathrm{C}\left(\mathrm{S}_{\mathrm{t}}, \mathrm{K}, \tau, \sigma, \mathrm{r}\right)=\mathrm{S}_{\mathrm{t}} \cdot \varphi\left(\mathrm{d}_{1}\right)-\mathrm{K} \cdot \mathrm{e}^{-\mathrm{r} \cdot \tau} \cdot \varphi\left(\mathrm{d}_{2}\right)
$$

Com:

$$
\mathrm{d}_{1}=\frac{\ln \left(\mathrm{S}_{\mathrm{t}} / \mathrm{K}\right)+\left(\mathrm{r}+\sigma^{2} / 2\right) \cdot \tau}{\sigma \cdot \sqrt{\tau}}
$$




$$
\mathrm{d}_{2}=\mathrm{d}_{1}-\sigma \cdot \sqrt{\tau}
$$

Sendo:

$\mathrm{C}\left(\mathrm{S}_{\mathrm{t}}, \mathrm{t},.\right)=$ Preço da opção de compra do ativo $\mathrm{S}$ no instante $\mathrm{t}$

$$
\varphi(.)=\text { função normal cumulativa padrão }\left(\varphi(x)=\frac{1}{\sqrt{2 \pi}} \cdot \int_{-\infty}^{x} \exp \left(z^{2} / 2\right) \cdot d z\right)
$$

$\mathrm{S}_{\mathrm{t}}=$ preço do ativo no instante $\mathrm{t}$

$\mathrm{K}$ = preço de exercício da opção (chamado também de strike)

$\tau=$ maturidade (tempo para o vencimento) da opção

$\sigma=$ volatilidade do ativo objeto

$\mathrm{r}=$ taxa de juros livre de risco

Diversas variações desta fórmula foram propostas. O modelo de Black (1976), que utilizaremos em nosso trabalho por ser o padrão de mercado para opções cambiais, é baseado nas mesmas premissas de Black-Scholes, com exceção de que não se assume que o preço spot do ativo segue um processo log-normal, mas sim que a densidade de probabilidade de seu preço futuro possui distribuição log-normal. Com estas premissas, o modelo determina o preço de uma opção de compra européia com a seguinte fórmula:

$$
\mathrm{C}\left(\mathrm{F}_{\mathrm{t}}, \mathrm{K}, \tau, \sigma, \mathrm{r}\right)=\mathrm{e}^{-\mathrm{r} \cdot \tau}\left[\mathrm{F}_{\mathrm{t}} \cdot \varphi\left(\mathrm{d}_{1}\right)-\mathrm{K} \cdot \varphi\left(\mathrm{d}_{2}\right)\right]
$$

Com: $\quad \mathrm{d}_{1}=\frac{\ln \left(\frac{\mathrm{F}_{\mathrm{t}}}{\mathrm{K}}\right)+\left(\frac{\sigma^{2}}{2}\right) \cdot \tau}{\sigma \sqrt{\tau}}$ e $\quad \mathrm{d}_{2}=\mathrm{d}_{1}-\sigma \sqrt{\tau}$

Sendo:

$\mathrm{C}()=$. preço da opção de compra

$\mathrm{F}_{\mathrm{t}}=$ preço futuro do ativo objeto

$\mathrm{K}$ = preço de exercício ou strike

$\mathrm{T}=$ data de exercício da opção 
$\mathrm{t}=$ instante de negociação

$\tau=\mathrm{T}-\mathrm{t}=$ maturidade

$\sigma=$ volatilidade do ativo objetivo

$\mathrm{r}=$ taxa de juros sem risco

$\varphi()=$. função normal cumulativa padrão.

De acordo com a paridade entre opções de compra e venda, o preço de uma opção de venda é obtida como:

$$
\mathrm{P}\left(\mathrm{F}_{\mathrm{t}}, \mathrm{K}, \tau, \sigma, \mathrm{r}\right)=\mathrm{e}^{-\mathrm{r \tau}} \cdot\left[\mathrm{K} \cdot \varphi\left(-\mathrm{d}_{2}\right)-\mathrm{F}_{\mathrm{t}} \varphi\left(-\mathrm{d}_{1}\right)\right]
$$

Uma outra abordagem para o apreçamento de opções, muito utilizado com opções americanas e exóticas é o modelo binomial inicialmente proposto por Cox; Ross e Rubinstein (1979). Os autores representam as possíveis variações do preço do ativo objeto como sendo movimentos discretos e em períodos discretos, em uma árvore binomial recombinante. Em cada "nó" da árvore, ou seja, um possível estado futuro do ativo objeto, seu preço pode subir por um fator $u(u>1)$ no instante seguinte $\left(\mathrm{S}_{\mathrm{t}+1}=\mathrm{S}_{\mathrm{t}}\right.$. $u$ ) com probabilidade $p$, ou cair por um fator $1 / u\left(\mathrm{~S}_{\mathrm{t}+1}=\mathrm{S}_{\mathrm{t}} / u\right)$ com probabilidade $(1-p)$. Os parâmetros $u$ e $p$ são ajustados de modo a refletir a volatilidade do ativo objeto.

O preço de uma opção é então obtido de modo regressivo: em cada nó da árvore, seu preço é calculado com base no preço desta mesma opção nos nós dos instantes seguintes:

$$
C_{t-\Delta t, i}=e^{-r \cdot \Delta t}\left[p \cdot C_{t, i+1}+(1-p) \cdot C_{t, i-1}\right]
$$

Com:

$\mathrm{C}_{\mathrm{t}, \mathrm{i}}=$ preço da opção no i-ésimo nó do instante $\mathrm{t}$

$\mathrm{p}=$ probabilidade de que o preço da opção suba no instante seguinte

$\mathrm{r}=$ juros livre de risco

$\Delta \mathrm{t}=$ tempo decorrido entre dois nós 


\subsection{Volatilidade Implícita e Efeito Smile}

De todas as variáveis que compõe as fórmulas de apreçamento baseadas no modelo de Black-Scholes, apenas a volatilidade não é diretamente observável, sendo uma variável de estado do preço do ativo objeto. Entende-se como volatilidade implícita no preço de uma opção o valor de volatilidade que aplicado na fórmula de apreçamento, iguale o resultado com o preço observado da opção $\left(\mathrm{C}_{\mathrm{obs}}\right.$ ou.P $\left.\mathrm{P}_{\mathrm{obs}}\right)$. Se não houvesse erro nas observações nem nas premissas do modelo de apreçamento, todas as opções negociadas no mesmo instante de tempo e sobre o mesmo ativo objeto deveriam apresentar a mesma volatilidade implícita.

Mas como documentado em diversos estudos, isto não ocorre. Em três trabalhos diferentes, Dupire (1994), Rubinstein (1994) e Derman e Kani (1994) analisaram o índice de ações S\&P500 (e alguns outros mercados como o índice Nikkey225) e observaram diferenças de volatilidade entre as diversas opções (acentuadas após a queda dos mercados de 1987), caracterizando o efeito smile, em que opções com strike muito distante do preço do ativo objeto possuem volatilidade implícita maior que as demais. Utilizando metodologias semelhantes, os três trabalhos utilizaram um modelo de árvore binomial recombinante para explicar as volatilidades implícitas observadas. Para isso, ajusta-se o modelo de Black-Scholes de modo a permitir que a volatilidade implícita fosse variável no tempo e em função do preço do ativo objeto:

$$
\mathrm{dS}_{\mathrm{t}}=\mu \cdot \mathrm{S}_{\mathrm{t}} \cdot \mathrm{dt}+\sigma\left(\mathrm{S}_{\mathrm{t}}, \mathrm{t}\right) \cdot \mathrm{S}_{\mathrm{t}} \cdot \mathrm{dW_{ \textrm {t } }}
$$

A partir da metodologia de árvores binomiais dos trabalhos de Dupire (1994), Rubinstein (1994) e Derman e Kani (1994), é possível extrair as volatilidades implícitas dos preços de opções observados no mercado. Todavia, estes trabalhos não expressam preocupação com a instabilidade numérica de seus métodos e a necessidade dos dados serem filtrados e regularizados (AVELLANEDA et al., 1997), assim, não adotaremos esta abordagem em nosso trabalho. 


\subsection{Cálculo da superfície de volatilidade}

Uma superfície de volatilidade implícita de opções é a representação em um dado instante de tempo, das volatilidades implícitas nos preços das opções negociadas no mercado naquele instante, em função das variáveis maturidade (o tempo para vencimento da opção) e moneyness (a razão entre o preço de exercício da opção e o preço do ativo objeto). A superfície de volatilidade implícita é, portanto, dada pela seguinte função:

$$
\sigma_{\mathrm{t}}^{\mathrm{imp}}\left(\mathrm{K} / \mathrm{S}_{\mathrm{t}}, \mathrm{T}-\mathrm{t}\right)=\sigma_{\mathrm{t}}^{\mathrm{imp}}(\mathrm{m}, \tau)
$$

Onde:

$$
\begin{aligned}
& \sigma_{\mathrm{t}}^{\mathrm{imp}}=\text { volatilidade implícita no instante } \mathrm{t} \\
& \mathrm{K}=\text { preço de exercício } \\
& \mathrm{S}_{\mathrm{t}}=\text { preço do ativo objeto no instante } \mathrm{t} \\
& \mathrm{T}=\text { data de exercício da opção } \\
& \mathrm{T}-\mathrm{t}=\text { maturidade }(\tau) \\
& \mathrm{K} / \mathrm{S}_{\mathrm{t}}=\text { moneyness }(\mathrm{m})
\end{aligned}
$$

Como as opções são negociadas apenas para alguns preços e datas de exercício, tornase necessário a interpolação das volatilidades implícitas observadas nestas opções para compor toda a superfície.

No caso das opções de dólar/real que estudaremos, há apenas opções negociadas com vencimentos nos primeiros dias úteis dos meses mais próximos e com preços de exercício discretizados em $\mathrm{R} \$ 0,05$ próximos ao preço do dólar spot. Como exemplo, mostramos as opções negociadas no dia 14/08/2006, quando o dólar spot era negociado à R\$2,1609: 


\begin{tabular}{|c|c|c|c|c|c|c|c|c|c|c|c|c|c|c|c|c|c|}
\hline & \multicolumn{17}{|c|}{ Preço de exercício } \\
\hline vencimento & 2,00 & 2,05 & 2,10 & 2,15 & 2,20 & 2,25 & 2,30 & 2,35 & 2,40 & 2,45 & 2,50 & 2,55 & 2,60 & 2,65 & 2,70 & 2,75 & 2,80 \\
\hline 1/9/2006 & & $x$ & $x$ & $\mathrm{x}$ & $x$ & $\mathrm{x}$ & $x$ & & & & & & & & & & \\
\hline $2 / 10 / 2006$ & & & & $\bar{x}$ & & $\bar{x}$ & $\bar{x}$ & & & & & & & & & & \\
\hline 1/11/2006 & & & & & & & & & & & & & & & & & \\
\hline 1/12/2006 & & & & & & & & & & & & & & & & & \\
\hline $2 / 1 / 2007$ & & & & & & $\mathrm{x}$ & & $\mathrm{x}$ & $\mathrm{x}$ & $\mathrm{x}$ & $\mathrm{x}$ & $x$ & $x$ & & $\mathrm{x}$ & & $x$ \\
\hline 1/2/2007 & & & & $x$ & & $x$ & & & $\bar{x}$ & & & & & & & & \\
\hline $1 / 3 / 2007$ & & & & & & & & & & & & & & & & & \\
\hline $2 / 4 / 2007$ & $x$ & $\mathrm{x}$ & $\mathrm{x}$ & & & & & & & & & & & & & & \\
\hline
\end{tabular}

Tabela 1 - opções negociadas em 14/ago/06

No dia seguinte, o dólar spot valia $\mathrm{R}$ \$2,1404 e as opções negociadas eram:

\begin{tabular}{|c|c|c|c|c|c|c|c|c|c|c|c|c|c|c|c|c|c|}
\hline & \multicolumn{17}{|c|}{ Preço de exercício } \\
\hline vencimento & 2,00 & 2,05 & 2,10 & 2,15 & 2,20 & 2,25 & 2,30 & 2,35 & 2,40 & 2,45 & 2,50 & 2,55 & 2,60 & 2,65 & 2,70 & 2,75 & 2,80 \\
\hline 1/9/2006 & & $x$ & $x$ & $x$ & $\mathrm{x}$ & $\mathrm{x}$ & $x$ & & & & & & & & & & \\
\hline 2/10/2006 & & & $x$ & $\bar{x}$ & $\bar{x}$ & & & & & & & & & & & & \\
\hline $1 / 11 / 2006$ & & & & & & $\mathrm{x}$ & & & & & & & & & & & \\
\hline $1 / 12 / 2006$ & & & & & & $x$ & & & & & & & & & & & \\
\hline 2/1/2007 & & & $\mathrm{x}$ & & $x$ & & & $x$ & $x$ & $x$ & $x$ & $x$ & $x$ & & $x$ & & $\mathrm{x}$ \\
\hline 1/2/2007 & & & & & & & & & & & & & & & & & \\
\hline $1 / 3 / 2007$ & & & & & & & & & & & & & & & & & \\
\hline $2 / 4 / 2007$ & $x$ & $\bar{x}$ & $x$ & & & & & & & & & & & & & & \\
\hline 1/5/2007 & & & & & & & & & & & & & & & & & \\
\hline 1/6/2007 & & & & & & & & & & & & & & & & & \\
\hline $2 / 7 / 2007$ & & & & & & & & $\mathrm{x}$ & & & & & & & & & \\
\hline
\end{tabular}

Tabela 2 - opções negociadas em 15/ago/06

Aqui, já observa-se que as opções negociadas não são as mesmas ao longo dos dias. Além deste fato, com a passagem de um dia útil e com a mudança do dólar spot, temos duas tabelas diferentes indicando os pontos observados na superfície de volatilidade (em termos de maturidade e moneyness) em cada um destes dias:

Para o dia $14 / 08 / 2006$

\begin{tabular}{|c|c|c|c|c|c|c|c|c|c|c|c|c|c|c|c|c|c|}
\hline & \multicolumn{17}{|c|}{ Moneyness } \\
\hline Maturidade (dias) & 0,93 & 0,95 & 0,97 & 0,99 & 1,02 & 1,04 & 1,06 & 1,09 & 1,11 & 1,13 & 1,16 & 1,18 & 1,20 & 1,23 & 1,25 & 1,27 & 1,30 \\
\hline 18 & & $x$ & $\bar{x}$ & $\mathrm{x}$ & $x$ & $x$ & $\bar{x}$ & & & & & & & & & & \\
\hline 49 & & & & $\mathrm{x}$ & & $\bar{x}$ & $\mathrm{x}$ & & & & & & & & & & \\
\hline 79 & & & & & & & & & & & & & & & & & \\
\hline 109 & & & & & & & & & & & & & & & & & \\
\hline 141 & & & & . & & $\mathrm{x}$ & & $x$ & $\mathrm{x}$ & $\bar{x}$ & $x$ & $x$ & $x$ & & $\bar{x}$ & & $\mathrm{x}$ \\
\hline 171 & & & & $x$ & & $\mathrm{x}$ & & & $\mathrm{x}$ & & & & & & & & \\
\hline 199 & & & & & & & & & & & & & & & & & \\
\hline 231 & $\bar{x}$ & $x$ & $\bar{x}$ & & & & & & & & & & & & & & \\
\hline 260 & & & & & & & & & & & & & & & & & \\
\hline 291 & & & & & & & & & & & & & & & & & \\
\hline 322 & & & & & & & & & & & & & & & & & \\
\hline
\end{tabular}

Tabela 3 - pontos da superfície de volatilidade em 14/ago/06 
Para o dia $15 / 08 / 2006$

\begin{tabular}{|c|c|c|c|c|c|c|c|c|c|c|c|c|c|c|c|c|c|}
\hline & \multicolumn{17}{|c|}{ Moneyness } \\
\hline Maturidade (dias) & 0,93 & 0,96 & 0,98 & 1,00 & 1,03 & 1,05 & 1,07 & 1,10 & 1,12 & 1,14 & 1,17 & 1,19 & 1,21 & 1,24 & 1,26 & 1,28 & 1,31 \\
\hline 17 & & $\mathrm{x}$ & $x$ & $\mathrm{x}$ & $\mathrm{x}$ & $\mathrm{x}$ & $\mathrm{x}$ & & & & & & & & & & \\
\hline 48 & & & $x$ & $x$ & $x$ & & & & & & & & & & & & \\
\hline 78 & & & & & & $\bar{x}$ & & & & & & & & & & & \\
\hline 108 & & & & & & $\mathrm{x}$ & & & & & & & & & & & \\
\hline 140 & & & $\mathrm{x}$ & & $\mathrm{x}$ & & & $\mathrm{x}$ & $x$ & $x$ & $x$ & $x$ & $x$ & & $x$ & & $x$ \\
\hline 170 & & & & & & & & & & & & & & & & & \\
\hline 198 & & & & & & & & & & & & & & & & & \\
\hline 230 & $x$ & $x$ & $x$ & & & & & & & & & & & & & & \\
\hline 259 & & & & & & & & & & & & & & & & & \\
\hline 290 & & & & & & & & & & & & & & & & & \\
\hline 321 & & & & & & & & $\mathrm{x}$ & & & & & & & & & \\
\hline
\end{tabular}

Tabela 4 - pontos da superfície de volatilidade em 15/ago/06

Dada esta diferença dos pontos observados a cada dia, vemos que é necessário a interpolação de uma superfície a cada dia de negócio para compararmos a variação das volatilidades implícitas no tempo, o que significa calcular a volatilidade implícita de opções não negociadas. A função de superfície de volatilidade resolve este problema, ao mapear a volatilidade implícita nas coordenadas desejadas de moneyness (a razão entre o preço de exercício da opção e o preço do ativo objeto) e maturidade.

Para o cálculo de superfície de volatilidade implícita, Aït-Sahalia e Lo (2000) utilizam o estimador de Kernel. Trata-se de um método não paramétrico de interpolação que faz uma média ponderada dos pontos observados em função da distância nas dimensões de moneyness e maturidade. Esta técnica também é utilizada por Cont e Fonseca (2002) e Fengler e Wang (2003), entre outros.

$$
\hat{\sigma}_{t}^{i m p}(m, \tau)=\frac{\sum_{i=1}^{L t} \sigma_{t}^{i m p}\left(m_{i, t}, \tau_{i, t}\right) \cdot g\left(\frac{m-m_{i, t}}{h_{m}}\right) \cdot g\left(\frac{\tau-\tau_{i, t}}{h_{\tau}}\right)}{\sum_{i=1}^{L t} g\left(\frac{m-m_{i, t}}{h_{m}}\right) \cdot g\left(\frac{\tau-\tau_{i, t}}{h_{\tau}}\right)}
$$

Com:

$\mathrm{m}=$ coordenada de moneyness (strike / valor do ativo) do ponto a ser interpolado $\tau=$ coordenada de maturidade do ponto a ser interpolado 
$\hat{\sigma}_{t}^{\text {imp }}(m, \tau)=$ volatilidade implícita estimada pela interpolação no tempo $t$ nas coordenadas $\{\mathrm{m}, \mathrm{\tau}\}$

$\mathrm{L}_{\mathrm{t}}=$ quantidade de opções observadas no dia $\mathrm{t}$

$\sigma_{\mathrm{t}}^{\mathrm{imp}}\left(\mathrm{m}_{\mathrm{i}, \mathrm{t}}, \tau_{\mathrm{i}, \mathrm{t}}\right)=$ volatilidade implícita da i-ésima opção observada no dia $\mathrm{t}$

$\mathrm{m}_{\mathrm{i}, \mathrm{t}}=$ moneyness da i-ésima opção observada no dia $\mathrm{t}$

$\tau_{\mathrm{i}, \mathrm{t}}=$ maturidade da i-ésima opção observada no dia $\mathrm{t}$

$\mathrm{h}_{\mathrm{m}}=$ ajuste de escala da dimensão moneyness

$\mathrm{h}_{\tau}=$ ajuste de escala da dimensão maturidade

$\mathrm{g}($.) $=$ função peso (função Kernel)

Aplicaremos também em nosso estudo, esta metodologia de estimador de Kernel para interpolação das volatilidades implícitas observadas em um dia de negócio. Nossa intenção é interpolar sempre os mesmos pontos, criando uma "matriz" padrão contendo os valores da superfície de volatilidade em pontos específicos, o que facilitará sua análise.

\subsection{Correlação entre a volatilidade implícita e o preço do ativo objeto}

Diversos trabalhos analisam a relação entre a variação das volatilidades implícitas no tempo e a variação do valor do ativo objeto.

Com a intenção de examinar a relação entre as variações das volatilidades implícitas e do ativo objeto, Skiadopoulos; Hodges e Clewlow (2000), organizaram as variações diárias das volatilidades implícitas das diversas opções observadas em um dia em faixas de diferentes maturidades (tempo para o vencimento).

As variáveis originais deste estudo são as diferenças diárias das volatilidades implícitas nos diversos níveis de moneyness (a razão entre o preço de exercício da opção e o preço do ativo objeto) observados em cada faixa de maturidade, obtendo assim $M$ variáveis aleatórias para cada uma destas faixas. 
Ao invés de analisar estas $M$ variáveis aleatórias os autores empregaram a técnica de análise de componentes principais. Com esta técnica, cria-se uma nova base no $\mathbf{R}^{\mathrm{N}}$ $(\mathrm{N}<\mathrm{M})$ de modo que a projeção das variáveis originais nesta nova base gera um novo conjunto de $\mathrm{N}$ variáveis aleatórias ortogonais. Estas novas variáveis ortogonais explicam a maior parte da variância do processo original:

$$
\begin{aligned}
& \mathrm{B}=\text { variáveis originais (dimensão }\{\mathrm{B}\}=\mathrm{M}) \\
& \mathrm{V}=\text { base do } \mathbf{R}^{\mathrm{N}}(\mathrm{N}<\mathrm{M}) \\
& \mathrm{Z}=\left\langle\mathrm{B}, \mathrm{V}>=\mathrm{E}\left(\mathrm{B}^{\prime} \cdot \mathrm{V}\right)=\text { novas variáveis, projeções das variáveis originais em } \mathrm{V}\right. \\
& \tilde{\mathrm{B}}=\mathrm{V} \cdot \mathrm{Z}=\text { aproximação de } \mathrm{B} \text { que minimiza o erro: } \mathrm{E}(\mathrm{B}-\tilde{\mathrm{B}})^{2}
\end{aligned}
$$

Em seguida, os autores analisam a correlação de cada variável obtida por este processo com a variação do preço futuro do ativo objeto (uma alternativa à análise da variação do preço do ativo objeto em si), o índice S\&P500. A análise é feita ano a ano, de 1992 a 1995 e embora as correlações sejam instáveis, variando a cada ano, é possível concluir que o nível de volatilidade implícita aumenta quando há queda no valor do ativo objeto.

Cont e Fonseca (2002) se propuseram a analisar a correlação entre as variações da superfície de volatilidade e as variações no valor do ativo objeto para dois mercados diferentes, o S\&P500 e FTSE1000. Os autores utilizaram o método não-paramétrico de interpolação de estimador de Kernel para criar a superfície e obter sua diferença. Esta diferença de um dia da superfície de volatilidade implícita é interpretada como uma realização de um campo aleatório bidimensional e representada pela função:

$\mathrm{B}(\omega$, maturidade, moneyness $)$

Sobre a variação diária desta superfície contínua, os autores aplicaram uma variante do método de análise de componentes principais e criaram $\mathrm{N}$ funções $\mathrm{V}_{\mathrm{i}}$, bidimensionais, ortogonais e invariantes, que formam uma base ortogonal para a representação da superfície. A seguir, cada realização do campo, ou superfície, aleatória B é aproximada pela seguinte decomposição: 
$\tilde{\mathrm{B}}(\omega$, maturidade, moneyness, $\mathrm{N})=\sum_{\mathrm{i}=1}^{\mathrm{N}} \mathrm{Z}_{\mathrm{i}}(\omega) \cdot \mathrm{V}_{\mathrm{i}}($ maturidade, moneyness $)$

Onde: $\mathrm{Z}=\langle\mathrm{B}, \mathrm{V}\rangle=\mathrm{E}\left(\mathrm{B}^{\prime} \cdot \mathrm{V}\right)$

Ou seja, $\mathrm{Z}_{\mathrm{i}}(\omega)$ são as variáveis aleatórias obtidas pela projeção de $\mathrm{B}\left(\omega\right.$, . ) em $\mathrm{V}_{\mathrm{i}}($.$) .$ Com este método, garante-se minimizar o erro quadrático: $\mathrm{E}(\mathrm{B}-\tilde{\mathrm{B}})^{2}$.

Por meio desta metodologia, os autores atestaram para dois mercados diferentes, o S\&P500 e FTSE1000, que há correlação razoavelmente significativa entre os parâmetros da decomposição da superfície de volatilidade (as variáveis $\mathrm{Z}_{\mathrm{i}}$ ) e a variação do valor ativo objeto: para a primeira variável, o fator de correlação chega a 0,7 no caso do FTSE1000 e a 0,66 no caso do S\&P500, sendo estas as correlações mais significativas.

Assim como nestes trabalhos, utilizaremos em nosso estudo o método de componentes principais para obter variáveis ortogonais representativas do processo de variação diária da superfície de volatilidade das opções de dólar. Deste modo, será possível avaliarmos a relação deste processo com as variações do preço do ativo objeto, no caso, o dólar spot.

\subsection{Comportamento da Superfície de Volatilidade ao longo do tempo}

Outra análise feita na literatura é sobre a regra empírica descrita por Derman (1999), em que a volatilidade implícita depende apenas da razão entre strike e preço do ativo objeto $(\mathrm{K} / \mathrm{S})$ e da maturidade. Ou seja, por esta regra, chamada de sticky-moneyness, a superfície de volatilidade implícita se mantém constante no tempo, fato que a maioria dos estudos prévios, em diversos mercados rejeita.

Na abordagem adotada por Dumas; Fleming e Whaley (1998) os autores modelaram as volatilidades implícitas observadas com funções paramétricas variáveis no tempo, como por exemplo: 


$$
\hat{\sigma}_{t}^{\text {imp }}(K, \tau)=\max \left(0,01 ; a_{0}(t)+a_{1}(t) \cdot K+a_{2}(t) \cdot K^{2}+a_{3}(t) \cdot \tau+a_{4}(t) \cdot K \cdot \tau\right)
$$

Com:

$$
\begin{aligned}
& \mathrm{K}=\text { Preço de exercício } \\
& \tau=\text { Maturidade } \\
& \hat{\sigma}_{\mathrm{t}}^{\mathrm{imp}}=\text { volatilidade implícita estimada pelo modelo paramétrico } \\
& \sigma_{\mathrm{t}}^{\mathrm{imp}}=\text { volatilidade implícitas observada } \\
& \mathrm{a}_{0}(\mathrm{t}), \ldots \mathrm{a}_{4}(\mathrm{t})=\text { parâmetros da função }
\end{aligned}
$$

A fórmula contém a função de máximo entre o polinômio e o valor 0,01 (1\% de volatilidade) para garantir que volatilidades negativas não são geradas. Os parâmetros $a(t)$ são então estimados de modo a reduzir o erro (diversos critérios de erro são utilizados) da função em relação às volatilidades implícitas observadas. Aplicando esta metodologia sobre o índice S\&P500, os autores concluíram que as volatilidades implícitas variam com o tempo e que a volatilidade estimada pelo modelo paramétrico em uma semana era consideravelmente diferente da volatilidade implícita observada na semana seguinte.

Em Alexander (2001), analisando o índice FTSE100, o autor aplica a metodologia de análise de componentes principais sobre as curvas de volatilidade (para uma maturidade fixa). São obtidos 3 componentes principais (novas variáveis) pela projeção das variáveis aleatórias originais sobre uma base de três dimensões:

$$
\begin{aligned}
& \mathrm{B}=\text { variáveis originais } \\
& \mathrm{V}=\text { base do } \mathbf{R}^{3} \\
& \mathrm{Z}=\langle\mathrm{B}, \mathrm{V}\rangle=\mathrm{E}\left(\mathrm{B}^{\prime} \cdot \mathrm{V}\right)=\text { novas variáveis, projeções das variáveis originais em } \mathrm{V}
\end{aligned}
$$

O trabalho conclui que as variáveis obtidas são sensíveis às variações do ativo objeto e que, portanto, a regra de sticky-moneyness, segundo a qual a superfície de volatilidade é fixa, não reflete a realidade.

Fengler; Härdle e Mammen (2005) estudaram o DAX (índice de ações alemão), e comprovaram que sua modelagem das variações da superfície de volatilidade no 
tempo é $10 \%$ mais precisa que a regra de sticky-moneyness, mostrando que a regra simplifica a realidade.

Em Leon e Oker (2005), analisando o mercado de opções de renda fixa em Euro, os autores mostram que a regra de sticky-moneyness é razoável, sendo que, na média, para um acompanhamento da superfície por 62 dias, a regra tem R-quadrado (coeficiente de determinação) de 0,65.

\subsection{Tratamento de dados analisados}

A diferença de tempo entre os negócios das opções e do ativo objeto e a falta de liquidez do mercado de opções fazem com que os preços observados não sejam necessariamente o preço justo de mercado. Como estas imperfeições de mercado podem gerar distorções nos estudos de volatilidade implícita, muitos autores eliminam algumas observações tidas como mais sensíveis às distorções no preço. Outro ponto importante na obtenção dos dados é qual deve ser o preço considerado das opções, se o preço de fechamento do pregão ou a média entre os preços de compra e venda. Listamos abaixo qual a metodologia de tratamento de dados que alguns trabalhos anteriores consideraram:

No estudo de Aït-Sahalia e Lo (2000), sobre as opções de européias do S\&P500, os autores consideraram a média entre os preços de compra e venda e eliminaram de sua amostra as opções com apenas 1 dia para o vencimento e preço menor que 0,125. Também excluíram do estudo opções cuja volatilidade implícita fosse maior que 0,7.

Em Skiadopoulos et al. (2000), as opções americanas do S\&P500 são analisadas pelo seu preço de fechamento e uma série de restrições nos dados foram consideradas, de modo que foram eliminadas: opções com maturidade abaixo de 10 dias, opções in-themoney, opções com a grega vega (a sensibilidade de seu preço em relação à volatilidade) abaixo de 8 e observações que levam à arbitragem. 
Os autores de Fengler e Wang (2003), em seu estudo sobre o DAX (índice de ações alemão) eliminaram as opções que levassem à volatilidade implícita acima de 0,7 ou abaixo de 0,1 .

Já em Cont e Fonseca (2002) são considerados no trabalho, sobre opções européias do S\&P500 e do FTSE100, os preços de fechamento de pregão e excluem-se valores de moneyness fora do intervalo [0,5 1,5] e maturidades fora do intervalo [1mês 1ano].

Em nosso estudo, dado que estamos sujeitos a erros de sincronismo (diferença temporal entre o último negócio realizado da opção e o último negócio do ativo objeto), uma vez que trabalhamos com preços de fechamento e há relativamente grande falta de liquidez no mercado de opções brasileiro, utilizaremos alguns dos tratamentos apresentados em trabalhos anteriores. 


\section{Capítulo 3}

\section{Análise de Componentes Principais}

Nesta seção, explicaremos a metodologia de análise de componentes principais (ver Cont e Fonseca (2002) e Meucci (2005)), que será aplicada na análise da superfície de volatilidade. Resumidamente esta metodologia consiste na transformação de $M$ variáveis aleatórias (ou então uma variável M-dimensional) possivelmente correlacionadas em um número menor de variáveis, descorrelacionadas.

\subsection{Metodologia da Análise de Componentes Principais}

Considere B uma variável aleatória com dimensão M de momento centrado zero, ou seja, de esperança nula. É possível decompor B do seguinte modo:

$$
\mathrm{B}=\mathrm{Q}^{1 / 2} \cdot \mathrm{U}
$$

Sendo U uma nova variável aleatória, também de dimensão $M$ com as seguintes propriedades:

$$
\begin{aligned}
& \mathrm{E}(\mathrm{U})=0 \quad \mathrm{e} \\
& \operatorname{Cov}(\mathrm{U})=\mathrm{E}\left(\mathrm{U}^{2}\right)=\mathrm{I}
\end{aligned}
$$

Neste caso, a matriz Q é a matriz de covariância de B, conforme a prova a seguir:

$$
\begin{aligned}
& \operatorname{cov}(\mathrm{B})=E\left(\mathrm{Q}^{1 / 2} \cdot \mathrm{U} \cdot \mathrm{U}^{\prime} \cdot \mathrm{Q}^{1 / 2}\right)= \\
& =\mathrm{Q}^{1 / 2} \cdot E\left(U \cdot U^{\prime}\right) \cdot \mathrm{Q}^{1 / 2}=\mathrm{Q}^{1 / 2} \cdot I \cdot \mathrm{Q}^{1 / 2}=\mathrm{Q}
\end{aligned}
$$

A matriz Q é, portanto, quadrada positiva definida, sobre a qual faremos a decomposição em autovalores e autovetores: 
Capítulo 3 - Análise de Componentes Principais

$$
\mathrm{Q}=\mathrm{L} \cdot \mathrm{D} \cdot \mathrm{L}^{\prime}
$$

A matriz $\mathrm{L}$ é composta pelos autovetores de $\mathrm{Q}=\left\{\mathrm{v}_{1}, \ldots \mathrm{v}_{\mathrm{M}}\right\}$ e $\mathrm{D}$ a matriz diagonal com os respectivos autovalores $=\left\{\lambda_{1}, \ldots, \lambda_{M}\right\}$ :

$$
\mathrm{L}=\left(\left[\mathrm{v}_{1}\right]\left[\mathrm{v}_{2}\right] \ldots\left[\mathrm{v}_{\mathrm{M}}\right]\right) ; \mathrm{D}=\left(\begin{array}{ccc}
\lambda_{1} & & 0 \\
& \ddots & \\
0 & & \lambda_{\mathrm{M}}
\end{array}\right)
$$

Os autovetores $v_{i}$ são ortonormais e compõe uma base para o $\mathbf{R}^{\mathrm{N}}$. Por sua ortonormalidade, temos que:

$$
\mathrm{v}_{\mathrm{i}} \cdot \mathrm{v}_{\mathrm{i}}=1 \quad \text { e } \quad \mathrm{v}_{\mathrm{i}}{ }^{\prime} \cdot \mathrm{v}_{\mathrm{j}}=0, \mathrm{i} \neq \mathrm{j}
$$

Assim, Q e B podem ser representados do seguinte modo:

$$
\begin{aligned}
& \mathrm{Q}=\sum_{\mathrm{i}=1}^{\mathrm{M}} \lambda_{\mathrm{i}} \cdot \mathrm{v}_{\mathrm{i}} \cdot \mathrm{v}_{\mathrm{i}}{ }^{\prime} \Rightarrow \mathrm{Q}^{1 / 2}=\sum_{\mathrm{i}=1}^{\mathrm{M}} \lambda_{\mathrm{i}}^{1 / 2} \cdot \mathrm{v}_{\mathrm{i}} \cdot \mathrm{v}_{\mathrm{i}}{ }^{\prime} \\
& \Rightarrow \mathrm{B}=\sum_{\mathrm{i}=1}^{\mathrm{M}} \lambda_{\mathrm{i}}^{1 / 2} \cdot \mathrm{v}_{\mathrm{i}} \cdot \mathrm{v}_{\mathrm{i}}{ }^{\prime} \cdot \mathrm{U}
\end{aligned}
$$

Fazendo $\hat{U}_{\mathrm{i}}$ a projeção de $\mathrm{U}$ no vetor $\mathrm{v}_{\mathrm{i}}$, ou seja: $\hat{U}_{\mathrm{i}}=\mathrm{v}_{\mathrm{i}} \cdot \mathrm{U}=\left\langle\mathrm{U}, \mathrm{v}_{\mathrm{i}}\right\rangle$, chegamos à:

$$
\begin{aligned}
& \mathrm{B}=\sum_{\mathrm{i}=1}^{\mathrm{M}} \lambda_{\mathrm{i}}^{1 / 2} \cdot \mathrm{v}_{\mathrm{i}} \cdot \hat{\mathrm{U}}_{\mathrm{i}} \\
& \Rightarrow \mathrm{B}=\sum_{\mathrm{i}=1}^{\mathrm{M}} \mathrm{z}_{\mathrm{i}} \cdot \mathrm{v}_{\mathrm{i}}
\end{aligned}
$$

Onde $\mathrm{Z}_{\mathrm{i}}=\lambda_{\mathrm{i}}^{1 / 2} \cdot \hat{\mathrm{U}}_{\mathrm{i}}$ é uma variável aleatória, projeção de $\mathrm{B}$ em $\mathrm{v}_{\mathrm{i}}$.

Algumas propriedades importantes de $\hat{U}_{\mathrm{i}}$ e $\mathrm{Z}_{\mathrm{i}}$ são:

$$
\begin{aligned}
& E\left(\hat{U}_{i}\right)=E\left(v_{i} \cdot U\right)=v_{i} \cdot E(U)=0 \\
& E\left(\hat{U}_{i}^{2}\right)=E\left(v_{i} \cdot U \cdot U^{\prime} \cdot v_{i}\right)=v_{i} \cdot E\left(U \cdot U^{\prime}\right) \cdot v_{i}=\left\|v_{i}\right\|^{2}=1 \\
& E\left(\hat{U}_{i} \cdot \hat{U}_{j}\right)=E\left(v_{i}^{\prime} \cdot U \cdot U^{\prime} \cdot v_{j}\right)=v_{i} \cdot E\left(U \cdot U^{\prime}\right) \cdot v_{j}=v_{i} \cdot v_{j}=0, i \neq j
\end{aligned}
$$




$$
\begin{aligned}
& E\left(z_{i}\right)=\lambda_{i}^{1 / 2} \cdot E\left(\hat{U}_{i}\right)=0 \\
& \operatorname{var}\left(z_{i}\right)=E\left(z_{i}^{2}\right)=\lambda_{i} \cdot E\left(\hat{U}_{i}^{2}\right)=\lambda_{i} \\
& \operatorname{cov}\left(z_{i}, z_{j}\right)=E\left(z_{i} \cdot z_{j}\right)=\lambda_{i}^{1 / 2} \cdot E\left(\hat{U}_{i} \cdot \hat{U}_{j}\right) \cdot \lambda_{j}^{1 / 2}=0 \quad, i \neq j
\end{aligned}
$$

Agora, na metodologia de componentes principais, pretende-se aproximar o processo B por um processo $\widetilde{B}(N)$, representação de $B$ em uma base de dimensão reduzida (dimensão $\mathrm{N})$, de modo a minimizar o erro quadrático: $\min \mathrm{E}\left(\|\mathrm{B}-\widetilde{\mathrm{B}}(\mathrm{N})\|^{2}\right)$

Assumindo que os autovetores e respectivos autovalores estão organizados de tal forma que $\lambda_{1}>\lambda_{2}>\ldots>\lambda_{M}$, temos que $v_{1}$ é a direção na qual a projeção de $B$ apresenta maior variância, $\mathrm{v}_{2}$ é a direção ortogonal a $\mathrm{v}_{1}$ na qual a projeção de $\mathrm{B}$ apresenta sua segunda maior variância e assim sucessivamente. Portanto, a base de dimensão $\mathrm{N}(\mathrm{N}<$ M) a ser escolhida para representar B de modo a minimizar $E\left(\|B-\tilde{B}(N)\|^{2}\right)$ é um subconjunto de $\left\{\mathrm{v}_{1}, \ldots \mathrm{v}_{\mathrm{M}}\right\}$, mais especificamente, um subconjunto composto dos $\mathrm{N}$ primeiros autovetores, conforme prova:

$$
\begin{aligned}
& \tilde{\mathrm{B}}(\mathrm{N})=\sum_{\mathrm{i} \in \mathrm{An}} \mathrm{z}_{\mathrm{i}} \cdot \mathrm{v}_{\mathrm{i}} \\
& \Rightarrow \mathrm{E}\left(\|\mathrm{B}-\tilde{\mathrm{B}}(\mathrm{N})\|^{2}\right)=\sum_{\mathrm{i} \in \mathrm{An}^{\mathrm{C}}} \mathrm{E}\left(\left\|\mathrm{z}_{\mathrm{i}} \cdot \mathrm{v}_{\mathrm{i}}\right\|^{2}\right)=\sum_{\mathrm{i} \in \mathrm{An}^{\mathrm{C}}} \mathrm{E}\left(\mathrm{z}_{\mathrm{i}}^{2}\right)=\sum_{\mathrm{i} \in \mathrm{An}^{\mathrm{C}}} \lambda_{\mathrm{i}}
\end{aligned}
$$

Onde An é o subconjunto dos índices $\{1, \ldots, \mathrm{M}\}$ com $\mathrm{N}$ elementos e $\mathrm{An}^{\mathrm{C}}$ é seu complemento. Portanto, de modo a minimizar o erro quadrático, $\mathrm{An}^{\mathrm{C}}$ deve conter os menores (e últimos) autovalores, ou seja, An deve conter os $\mathrm{N}$ maiores (e primeiros) autovalores. Portanto:

$$
\tilde{\mathrm{B}}(\mathrm{N})=\sum_{\mathrm{i}=1}^{\mathrm{N}} \mathrm{z}_{\mathrm{i}} \cdot \mathrm{v}_{\mathrm{i}}
$$

Outro modo de abordar este problema é minimizar a dimensão N, com a restrição de um R-quadrado mínimo de $\widetilde{\mathrm{B}}(\mathrm{N})$ em relação à $\mathrm{B}$. Este é um valor dado, que representaremos por $\mathrm{R}_{\min }^{2}$. Partimos da definição do R-quadrado generalizado entre 
duas variáveis (MEUCCI, 2005), medida do seu grau de correspondência e restringimos seu resultado para ser maior ou igual ao valor dado $\mathrm{R}_{\min }^{2}$ :

$$
\begin{aligned}
& \mathrm{R}^{2}\{\mathrm{~B}, \widetilde{\mathrm{B}}(\mathrm{N})\} \equiv 1-\frac{\mathrm{E}\left((\mathrm{B}-\tilde{\mathrm{B}}(\mathrm{N}))^{\prime} \cdot(\mathrm{B}-\tilde{\mathrm{B}}(\mathrm{N}))\right)}{\operatorname{tr}\{\operatorname{cov}(\mathrm{B})\}} \geq \mathrm{R}_{\min }^{2} \\
& \Rightarrow \mathrm{E}\left((\mathrm{B}-\tilde{\mathrm{B}}(\mathrm{N}))^{\prime} \cdot(\mathrm{B}-\tilde{\mathrm{B}}(\mathrm{N}))\right) \leq\left(1-\mathrm{R}_{\min }^{2}\right) \cdot \operatorname{tr}\{\operatorname{cov}(\mathrm{B})\} \\
& \Rightarrow \sum_{\mathrm{i} \in \mathrm{An}^{\mathrm{C}}} \lambda_{\mathrm{i}} \leq\left(1-\mathrm{R}_{\min }^{2}\right) \cdot \sum_{\mathrm{i}=1}^{\mathrm{M}} \lambda_{\mathrm{i}} \Rightarrow \frac{\sum_{\mathrm{i} \in \mathrm{An}}^{\mathrm{M}} \lambda_{\mathrm{i}}}{\sum_{\mathrm{i}=1}^{\mathrm{M}} \lambda_{\mathrm{i}}} \geq \mathrm{R}_{\min }^{2}
\end{aligned}
$$

Com:

$\operatorname{tr}\{\}=$. função traço, a soma dos elementos da diagonal principal de uma matriz quadrada

Assim An deve conter os maiores autovalores em quantidade que possa garantir um Rquadrado mínimo de B e $\widetilde{B}(\mathrm{~N})$.

\subsection{Correlação entre as componentes principais e uma variável aleatória uni-dimensional}

Agora queremos analisar qual a relação entre uma nova variável aleatória unidimensional centrada, y em relação à $\widetilde{\mathrm{B}}(\mathrm{N})$ (representação de $\mathrm{B}$ em dimensão reduzida $\mathrm{N})$ :

A métrica que adotaremos para esta análise é a covariância normalizada (mesmo princípio da correlação) entre $\widetilde{\mathrm{B}}(\mathrm{N})$ e $\mathrm{y}$ :

$$
\frac{\|\operatorname{cov}(\tilde{\mathrm{B}}(\mathrm{N}), \mathrm{y})\|}{\| \sqrt{\operatorname{var}(\tilde{\mathrm{B}}(\mathrm{N}))}) \cdot \| \sqrt{\operatorname{var}(\mathrm{y}) \|}}
$$

Como: 
Capítulo 3 - Análise de Componentes Principais

$$
\sqrt{\|\operatorname{var}(\tilde{\mathrm{B}}(\mathrm{N}))\|}=\sum_{\mathrm{i}=1}^{\mathrm{N}} \sqrt{\mathrm{E}\left(\left(\mathrm{z}_{\mathrm{i}}\right)^{2}\right)} \cdot\left\|\mathrm{v}_{\mathrm{i}}\right\|=\sqrt{\sum_{\mathrm{i}=1}^{\mathrm{N}} \lambda_{\mathrm{i}}}
$$

e:

$$
\begin{aligned}
& \operatorname{cov}(\widetilde{B}(N), y)=E(\widetilde{B}(N) \cdot y)=\sum_{i=1}^{N} E\left(z_{i} \cdot y\right) \cdot v_{i} \\
& \Rightarrow\|\operatorname{cov}(\widetilde{B}(N), y)\|=\left\|\sum_{i=1}^{N} E\left(z_{i} \cdot y\right) \cdot v_{i}\right\|=\sum_{i=1}^{N}\left|E\left(z_{i} \cdot y\right)\right|
\end{aligned}
$$

podemos reescrever, portanto, esta métrica como sendo:

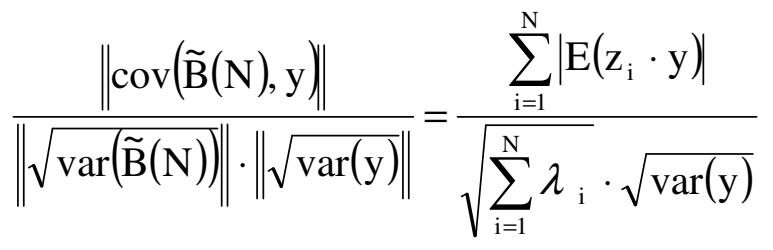

Esta é a métrica que utilizaremos para analisar a relação entre as variações da superfície de volatilidade implícita e as variações do valor do ativo objeto ao longo dos dias de negócio. Sua aplicação prática e também a aplicação da análise de componentes principais são descritas no capítulo seguinte. 


\section{Capítulo 4}

\section{Metodologia}

Apresentaremos neste capítulo a abordagem que será utilizada no estudo das volatilidades implícitas das opções de dólar/real. Faremos uso de metodologias já utilizadas em outros trabalhos, demonstradas na revisão bibliográfica.

Com o objetivo de entendermos o comportamento da superfície de volatilidade das opções de dólar/real ao longo do tempo, iremos estudar duas propriedades já analisadas pela literatura em superfícies de volatilidade de outros mercados (DAX, S\&P e FTSE) e comparar como seus resultados se refletem para as opções de dólar/real. As duas propriedades são:

\section{1) Regra de Sticky-Moneyness}

Uma das propriedades comumente estudada (e refutada) é a regra heurística normalmente considerada pelo mercado de sticky-moneyness, apresentada por Derman (1999), segundo a qual a volatilidade é constante no tempo para níveis de moneyness (relação entre o preço de strike e o preço do ativo $=\mathrm{K} / \mathrm{S}$ ) e maturidade (o tempo para o vencimento da opção). Por esta regra, portanto, a superfície de volatilidade implícita é invariável no tempo.

2) A correlação entre a variação da superfície de volatilidade e $\Delta \mathrm{S}$ : Outros estudos mostram, para diferentes mercados, que a variação diária da superfície é correlacionada com a variação do preço do ativo objeto, $\Delta \mathrm{S}$. Nestes estudos, as volatilidades implícitas são modeladas de modo a se obter séries temporais que representam a variação da superfície de volatilidade. 
Aplicaremos nosso estudo às opções européias em reais sobre o dólar comercial negociadas pela $B M \& F$, que possuem vários strikes e vencimentos, normalmente os primeiros dias úteis dos meses mais próximos à data de negociação, havendo, em média, 23 pontos por dia a partir dos quais a superfície do dia deve ser montada.

Para alcançar este objetivo, o trabalho a ser desenvolvido possui as seguintes etapas, que serão descritas com maior detalhe nas seções seguintes:

1 - Obtenção das volatilidades implícitas das opções de dólar negociadas ao longo do tempo.

2 - Construção das superfícies de volatilidade de cada dia por meio da interpolação das volatilidades implícitas obtidas na etapa 1.

3 - Decomposição da variação diária das superfícies de volatilidades implícitas $\left(\hat{\sigma}_{\mathrm{t}+1}^{\mathrm{imp}}(\mathrm{m}, \tau)-\hat{\sigma}_{\mathrm{t}}^{\mathrm{imp}}(\mathrm{m}, \tau)\right)$ em componentes principais, obtendo-se variáveis aleatórias que representam esta variação da superfície em uma nova base.

4 - Análise das correlações entre $\Delta S(t)$, a variação de preço do ativo objeto, e as variáveis que representam a superfície de volatilidade, obtidas na etapa 3.

5 - Análise estatística da variância da superfície de volatilidade.

\subsection{Cálculo das Volatilidades Implícitas}

Para o cálculo das volatilidades implícitas, vamos utilizar uma variação do modelo de Black-Scholes, o modelo de Black (1976) utilizado no mercado para o apreçamento das opções de dólar, que se baseia nas premissas do modelo de Black-Scholes, mas trabalha com o preço futuro do ativo objeto, evitando assim o problema de definir qual a taxa de juros livre de risco do dólar. Para facilitar o acompanhamento deste texto, replicamos as fórmulas de Black para apreçar opções de compra e venda (respectivamente $\mathrm{C}($.) e $\mathrm{P}($.$) ) já expostas no capítulo 2$ (fórmulas (2.3) e (2.4)): 


$$
\begin{aligned}
& \mathrm{C}\left(\mathrm{F}_{\mathrm{t}}, \mathrm{K}, \tau, \sigma, \mathrm{r}\right)=\mathrm{e}^{-\mathrm{r} \cdot \tau}\left[\mathrm{F}_{\mathrm{t}} \cdot \varphi\left(\mathrm{d}_{1}\right)-\mathrm{K} \cdot \varphi\left(\mathrm{d}_{2}\right)\right] \\
& \mathrm{P}\left(\mathrm{F}_{\mathrm{t}}, \mathrm{K}, \tau, \sigma, \mathrm{r}\right)=\mathrm{e}^{-\mathrm{r \tau} \tau} \cdot\left[\mathrm{K} \cdot \varphi\left(-\mathrm{d}_{2}\right)-\mathrm{F}_{\mathrm{t}} \varphi\left(-\mathrm{d}_{1}\right)\right]
\end{aligned}
$$

Queremos inverter estas fórmulas e obter as volatilidades em função do preço das opções observadas, ou seja, obter a volatilidade implícita. Isso é feito numericamente por meio do método de Newton-Raphson sobre a função $\mathrm{f}(\sigma)=\mathrm{C}(\sigma)-\mathrm{C}_{\mathrm{obs}}$, (no caso de opções de compra). O método estima a raiz de uma função a partir de iterações que são repetidas até alcançar-se uma precisão adequada. A fórmula iterativa é:

$$
\sigma_{i+1}=\sigma_{i}-\frac{f\left(\sigma_{i}\right)}{\partial f\left(\sigma_{i}\right) / \partial \sigma_{i}}
$$

Para o caso de $\mathrm{f}(\sigma)=\mathrm{C}(\sigma)-\mathrm{C}_{\mathrm{obs}}$ temos:

$$
\sigma_{i+1}=\sigma_{i}-\frac{f\left(\sigma_{i}\right)}{\partial f\left(\sigma_{i}\right) / \partial \sigma_{i}}=\sigma_{i}-\frac{\mathrm{C}\left(\sigma_{i}\right)-\mathrm{C}_{\mathrm{OBS}}}{\partial \mathrm{C}\left(\sigma_{\mathrm{i}}\right) / \partial \sigma_{\mathrm{i}}}=\sigma_{\mathrm{i}}-\frac{\mathrm{C}\left(\sigma_{\mathrm{i}}\right)-\mathrm{C}_{\mathrm{OBS}}}{\mathrm{Fe}^{-\mathrm{r \tau}} \cdot \varphi(\mathrm{d} 1) \cdot \sqrt{\tau}}
$$

Com:

$$
\begin{aligned}
& \text { i = quantidade de iterações já realizadas } \\
& \sigma_{\mathrm{i}}=\text { volatilidade implícita na i-ésima iteração do método } \\
& \mathrm{f}\left(\sigma_{\mathrm{i}}\right)=\mathrm{C}(\sigma)-\mathrm{C}_{\mathrm{obs}} \\
& \mathrm{C}\left(\sigma_{\mathrm{i}}\right)=\text { preço da opção segundo a fórmula Black-76 com volatilidade } \sigma_{\mathrm{i}} \\
& \mathrm{C}_{\mathrm{OBS}}=\text { preço observado da opção }
\end{aligned}
$$

A fórmula é a mesma para opções de venda. Agora, com as volatilidades implícitas das opções negociadas calculadas, prosseguiremos com a metodologia para obter a superfície de volatilidade.

\subsection{Interpolação da Superfície de Volatilidade}

Com os pontos observados de volatilidades implícitas, o próximo passo é a construção das superfícies de volatilidade de cada dia a partir de uma função de interpolação. 
Trata-se de uma representação da volatilidade em função das dimensões moneyness e maturidade: $\sigma_{\mathrm{t}}^{\mathrm{imp}}(\mathrm{m}, \tau)$.

Utilizaremos o método de interpolação como uma média ponderada dos pontos observados em função da distância nas dimensões de moneyness e maturidade. Tratase da técnica não paramétrica de estimador de Kernel, utilizada por Aït-Sahalia e Lo (2000), Cont e Fonseca (2002) e Fengler e Wang (2003), entre outros.

Nesta abordagem, dada uma coordenada $\{m, \tau\}$ qualquer, em um momento t, a volatilidade implícita a ela associada é a média das volatilidades implícitas das $\mathrm{L}_{\mathrm{t}}$ opções observadas no dia $\mathrm{t}: \sigma_{\mathrm{t}}^{\mathrm{imp}}\left(\mathrm{m}_{1, \mathrm{t}}, \tau_{1, \mathrm{t}}\right), \ldots, \sigma_{\mathrm{t}}^{\mathrm{imp}}\left(\mathrm{m}_{\mathrm{L}_{\mathrm{t}}, \mathrm{t}}, \tau_{\mathrm{L}_{\mathrm{t}}, \mathrm{t}}\right)$ ponderadas por uma função peso (chamada de função Kernel) que tem como entrada a distância entre a coordenada $\left\{\mathrm{m}_{\mathrm{i}, \mathrm{t}}, \tau_{\mathrm{i}, \mathrm{t}}\right\}$ de cada volatilidade implícita observada e a coordenada $\{\mathrm{m}, \tau\}$ do ponto a ser interpolado. A fórmula é a mesma apresentada no capítulo 2 (fórmula (2.8)) e a reapresentaremos aqui:

$$
\hat{\sigma}_{t}^{\text {imp }}(m, \tau)=\frac{\sum_{i=1}^{L t} \sigma_{t}^{i m p}\left(m_{i, t}, \tau_{i, t}\right) \cdot g\left(\frac{m-m_{i, t}}{h_{m}}\right) \cdot g\left(\frac{\tau-\tau_{i, t}}{h_{\tau}}\right)}{\sum_{i=1}^{L t} g\left(\frac{m-m_{i, t}}{h_{m}}\right) \cdot g\left(\frac{\tau-\tau_{i, t}}{h_{\tau}}\right)}
$$

Em nosso estudo, utilizamos a função gaussiana: $\mathrm{g}(\mathrm{u})=(2 \pi)^{-0.5} \exp \left(-\mathrm{u}^{2} / 2\right)$, como em Cont e Fonseca (2002). Mas, conforme observado por Aït-Sahalia e Lo (2000), Cont e Fonseca (2002) e Fengler e Wang (2003) os fatores $h_{\tau}$ e $h_{m}$ determinam o nível de suavização do método em cada uma das coordenadas e sua escolha é mais importante que a definição da função peso.

Como trabalharemos no domínio discreto, iremos interpolar a volatilidade implícita apenas para algumas coordenadas de moneyness e maturidade específicas, sobre as quais se dará a sequência do estudo. Com isto, resolvemos o problema das opções negociadas em dias consecutivos não possuírem as mesmas coordenadas de moneyness e maturidade (como observado na seção 2.3). 
Teremos portanto um conjunto de coordenadas de moneyness, com Lm pontos, $\left\{\mathrm{m}_{1}\right.$, $\left.\mathrm{m}_{2}, \ldots, \mathrm{m}_{\mathrm{Lm}}\right\}$, e um conjunto de coordenadas de maturidade com $\mathrm{L} \tau$ pontos, $\left\{\tau_{1}, \tau_{2}, \ldots\right.$, $\left.\tau_{\mathrm{L} \tau}\right\}$ sobre as quais trabalharemos em todos os dias estudados. Chamaremos de $\mathrm{M}$ a quantidade total de pontos estudados na superfície, dado por $\operatorname{Lm} x \mathrm{~L} \tau$.

Para cada dia observado temos a seguinte matriz de volatilidades implícitas interpoladas com dimensão $\mathrm{Lm}$ x L $\tau$ :

$$
\hat{\sigma}_{t}^{i m p}=\left[\begin{array}{ccc}
\hat{\sigma}_{t}^{\mathrm{imp}}\left(\mathrm{m}_{1}, \tau_{1}\right) & \cdots & \hat{\sigma}_{\mathrm{t}}^{\mathrm{imp}}\left(\mathrm{m}_{1}, \tau_{\mathrm{L} \tau}\right) \\
\vdots & \ddots & \vdots \\
\hat{\sigma}_{\mathrm{t}}^{\mathrm{imp}}\left(\mathrm{m}_{\mathrm{Lm}}, \tau_{1}\right) & \cdots & \hat{\sigma}_{\mathrm{t}}^{\mathrm{imp}}\left(\mathrm{m}_{\mathrm{Lm}}, \tau_{\mathrm{L} \tau}\right)
\end{array}\right]
$$

Para facilitar a manipulação numérica que se segue, trabalharemos com os dados rearranjando-os em um único vetor coluna de dimensão M:

$$
\hat{\sigma}_{t}^{\text {imp }}=\left[\begin{array}{c}
\hat{\sigma}_{t}^{\mathrm{imp}}\left(\mathrm{m}_{1}, \tau_{1}\right) \\
\hat{\sigma}_{\mathrm{t}}^{\mathrm{imp}}\left(\mathrm{m}_{1}, \tau_{2}\right) \\
\vdots \\
\hat{\sigma}_{\mathrm{t}}^{\mathrm{imp}}\left(\mathrm{m}_{1}, \tau_{\mathrm{L} \tau}\right) \\
\hat{\sigma}_{\mathrm{t}}^{\mathrm{imp}}\left(\mathrm{m}_{2}, \tau_{1}\right) \\
\vdots \\
\hat{\sigma}_{\mathrm{t}}^{\mathrm{imp}}\left(\mathrm{m}_{\mathrm{Lm}}, \tau_{\mathrm{L} \tau}\right)
\end{array}\right]
$$

\subsection{Decomposição da variação diária da superfície}

Agora, com a superfície de volatilidade construída, iremos utilizar o método de Análise de Componentes Principais, já apresentado.

Num dia t, observamos a variação da superfície de volatilidade implícita nos M pontos interpolados, dados pelo vetor coluna $\Delta \hat{\sigma}_{t}^{\text {imp }}$ :

$$
\Delta \sigma_{t}^{\mathrm{imp}}=\hat{\sigma}_{\mathrm{t}+1}^{\mathrm{imp}}-\hat{\sigma}_{\mathrm{t}}^{\mathrm{imp}}
$$


Vamos chamar de $\Delta \sigma^{\text {imp }}$ a variável aleatória que representa este processo observado de variação da superfície de volatilidade. A partir desta variável, criamos outra variável aleatória de dimensão M e média nula:

$$
\mathrm{B}=\Delta \sigma^{\mathrm{imp}}-\mathrm{E}\left(\Delta \sigma^{\mathrm{imp}}\right)
$$

Chamaremos de $\beta$ a matriz de dimensões $\mathrm{M}$ x (T-1), contendo as $\mathrm{T}-1$ realizações observadas da variável aleatória B M-dimensional. Obtemos $\beta$ pela fórmula:

$$
\left.\beta=\left(\left[\Delta \sigma_{1}^{\mathrm{imp}}\right] \Delta \sigma_{2}^{\mathrm{imp}}\right] \ldots\left[\Delta \sigma_{\mathrm{T}-1}^{\mathrm{imp}}\right]\right)-\frac{1}{\mathrm{~T}-1} \cdot\left(\sum_{\mathrm{t}=1}^{\mathrm{T}-1}\left[\Delta \sigma_{\mathrm{t}}^{\mathrm{imp}}\right]\right) \cdot \mathrm{e}_{\mathrm{T}-1}
$$

Nesta fórmula e $\mathrm{e}_{\mathrm{T}-1}$ é o vetor linha, de dimensão T-1, composto apenas de 1:

$$
\mathrm{e}_{\mathrm{T}-1}=[1,1, \ldots, 1]
$$

Conforme a metodologia de análise de componentes principais, obteremos os autovetores e autovalores da matriz de covariância de B. A matriz de covariância, Q é estimada a partir de $\beta$ :

$$
\mathrm{Q}=\operatorname{cov}(\mathrm{B})=\beta \cdot \beta^{\prime} /((\mathrm{T}-1)-1)
$$

Os autovetores e respectivos autovalores de Q são obtidos com a decomposição matricial, conforme a fórmula (3.3):

$$
\mathrm{Q}=\mathrm{L} \cdot \mathrm{D} \cdot \mathrm{L}^{\prime}
$$

A matriz L é composta pelos $M$ autovetores (de dimensão $M$ ) de $Q=\left\{v_{1}, \ldots v_{M}\right\}$ e $D$ é a matriz diagonal com os respectivos autovalores $=\left\{\lambda_{1}, \ldots, \lambda_{M}\right\}$ :

$$
\mathrm{L}=\left(\left[\mathrm{v}_{1}\right]\left[\mathrm{v}_{2}\right] \cdots\left[\mathrm{v}_{\mathrm{M}}\right]\right) ; \mathrm{D}=\left(\begin{array}{ccc}
\lambda_{1} & & 0 \\
& \ddots & \\
0 & & \lambda_{\mathrm{M}}
\end{array}\right)
$$

As matrizes são construídas de modo que $\lambda_{1}>\lambda_{2}>\ldots>\lambda_{M}$. Assim, os primeiros autovetores representam as direções do $\mathbf{R}^{\mathrm{M}}$ em que o processo $\mathrm{B}$ apresenta maior 
variância. Os autovetores são ortonormais, possuindo as seguintes propriedades já apresentadas no capítulo 3:

$$
\begin{aligned}
& \mathrm{v}_{\mathrm{i}}{ }^{\prime} \cdot \mathrm{v}_{\mathrm{i}}=1 \\
& \mathrm{v}_{\mathrm{i}}{ }^{\prime} \cdot \mathrm{v}_{\mathrm{j}}=0 \quad, \mathrm{i} \neq \mathrm{j}
\end{aligned}
$$

Seguindo a metodologia de análise de componentes principais, devemos escolher os autovetores a serem utilizados como base para representar B, processo centrado da variação da superfície de volatilidade. Iremos escolher os $\mathrm{N}$ primeiros autovetores de modo a garantir que a aproximação de $\mathrm{B}$ baseada nestes autovetores possua um Rquadrado mínimo ( $\mathrm{R}_{\min }^{2}$ ) dado. Reescrevendo a fórmula (3.12), escolhemos $\mathrm{N}$ tal que:

$$
\frac{\sum_{i=1}^{N} \lambda_{i}}{\sum_{i=1}^{M} \lambda_{i}} \geq R_{\text {min }}^{2}
$$

A aproximação do processo $B$ feita pela base $N$-dimensional $\left\{\mathrm{v}_{1}, \ldots \mathrm{v}_{\mathrm{N}}\right\}$ é dada pela fórmula (3.10) mostrada no capítulo 3, que apresentamos novamente:

$$
\tilde{\mathrm{B}}(\mathrm{N})=\sum_{\mathrm{i}=1}^{\mathrm{N}} \mathrm{z}_{\mathrm{i}} \cdot \mathrm{v}_{\mathrm{i}}
$$

Onde:

$$
\mathrm{z}_{\mathrm{i}}=\left\langle\mathrm{v}_{\mathrm{i}}, \mathrm{B}\right\rangle=\text { projeção ortogonal de B em } \mathrm{v}_{\mathrm{i}}
$$

Aqui $\left\{z_{1}, \ldots z_{N}\right\}$ são as novas variáveis obtidas a partir desta mudança de base, projeções de $B$ em $v_{i}$. As T-1 realizações de $z_{i}$ serão representadas pelas variáveis $\zeta_{i}$ (zeta), obtidas do seguinte modo:

$$
\zeta_{\mathrm{i}}=\mathrm{v}_{\mathrm{i}} \cdot \beta
$$

Com:

$$
\begin{aligned}
& \zeta_{\mathrm{i}}=\text { vetor-linha com as } \mathrm{T}-1 \text { realizações da variável } \mathrm{z}_{\mathrm{i}} \\
& \beta=\text { matriz } \mathrm{M} \times(\mathrm{T}-1) \text { com as realizações observadas de } \mathrm{B}
\end{aligned}
$$

Portanto, a variação centrada da superfície de volatilidade em um dia t qualquer pode ser representada como: 


$$
\tilde{\beta}_{t}(N)=\sum_{i=1}^{N} \zeta_{i}(t) \cdot v_{i}
$$

Com:

$\tilde{\beta}_{\mathrm{t}}(\mathrm{N})=$ vetor $\mathrm{M}$-dimensional representando a variação centrada da superfície de volatilidade implícita

$\zeta_{\mathrm{i}}(\mathrm{t})=$ realização da variável $\mathrm{z}_{\mathrm{i}}$ no instante $\mathrm{t}$

$\mathrm{v}_{\mathrm{i}}=\mathrm{i}$-ésimo autovetor de $\mathrm{B}$

A partir de $\widetilde{B}(N)$, representação $N$-dimensional da variação centrada da superfície de volatilidade implícita, iremos calcular qual a relação deste processo com a variação do valor do ativo objeto.

\subsection{Análise da correlação entre as variações da superfície de volatilidade e as variações do valor do ativo objeto:}

Lembramos que um de nossos objetivos é estudar se há correlação entre os movimentos da superfície e a variação do ativo objeto, o dólar spot. A metodologia para esta análise é apresentada a seguir.

Dado o espaço de probabilidades padrão $(\Omega, F, P)$, a cada dia t teremos uma realização $\omega$ da variação da superfície de volatilidade implícita que, pela metodologia exposta na seção anterior, é representada pela variável aleatória $\mathrm{N}$-dimensional $\widetilde{\mathrm{B}}(\mathrm{N})$. Também teremos, a cada dia, uma realização da variação do dólar spot, $\Delta \mathrm{S}$.

Para verificar a correlação entre as variáves, também trabalharemos com a variação centrada do dólar spot:

$$
\Delta \mathrm{S}_{\mathrm{c}}=\Delta \mathrm{S}-\Delta \overline{\mathrm{S}}
$$

Sendo: 
$\Delta \mathrm{S}$ = variável aleatória da variação diária do dólar spot

$\Delta \overline{\mathrm{S}}=$ esperança da variação do dólar spot. É estimada por: $\Delta \overline{\mathrm{S}}=\frac{1}{\mathrm{~T}-1} \cdot \sum_{\mathrm{i}=1}^{\mathrm{T}-1} \Delta \mathrm{S}_{\mathrm{i}}$

Nossa análise será a covariância normalizada de $\Delta \mathrm{S}_{\mathrm{c}}$ com $\widetilde{\mathrm{B}}(\mathrm{N})$. Conforme analisado anteriormente, baseado na fórmula (3.17), esta métrica pode ser escrita como:

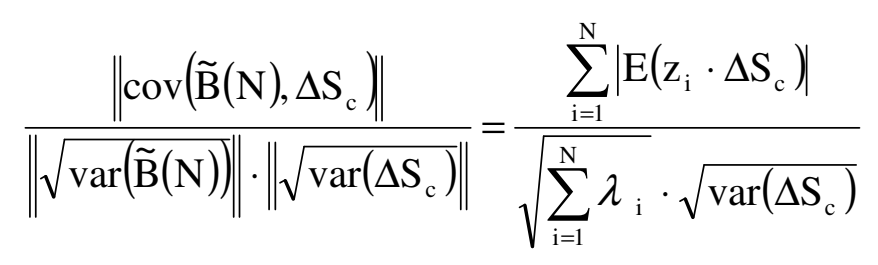

Com:

$\mathrm{Z}_{\mathrm{i}}=$ variável aleatória obtida pela projeção de $\mathrm{B}$ no vetor $\mathrm{v}_{\mathrm{i}}$

$\lambda_{\mathrm{i}}=$ autovalor referente ao vetor $\mathrm{v}_{\mathrm{i}}, \mathrm{i}$-ésimo autovetor de $\mathrm{Q}(\mathrm{Q}=\operatorname{cov}(\mathrm{B}))$

Os estimadores utilizados são:

$$
\mathrm{E}\left(\mathrm{z}_{\mathrm{i}} \cdot \Delta \mathrm{S}_{\mathrm{c}}\right)=\zeta_{\mathrm{i}} \cdot \Delta \mathrm{S}_{\mathrm{c}}^{\mathrm{R}} /((\mathrm{T}-1)-1)
$$

Com:

$\zeta_{\mathrm{i}}=$ vetor-linha com as $\mathrm{T}-1$ realizações da variável $\mathrm{z}_{\mathrm{i}}$

$\Delta \mathrm{S}_{\mathrm{C}}^{\mathrm{R}}=$ vetor-coluna com as $\mathrm{T}-1$ realizações da variável aleatória $\Delta \mathrm{S}_{\mathrm{c}}$

e:

$$
\operatorname{var}\left(\Delta \mathrm{S}_{\mathrm{c}}\right)=\Delta \mathrm{S}_{\mathrm{c}}^{\mathrm{R}} \cdot \Delta \mathrm{S}_{\mathrm{c}}^{\mathrm{R}} /((\mathrm{T}-1)-1)
$$

A covariância normalizada é um índice que varia de 0 à 1, indicando qual a relação linear entre $\widetilde{\mathrm{B}}(\mathrm{N})$ e $\Delta \mathrm{S}_{\mathrm{c}}$. Se obtivermos um índice próximo a 1 , isso significa que as duas variáveis são correlacionadas. Inversamente, caso o índice estiver próximo a 0, podemos concluir que as variáveis são descorrelacionadas. 


\subsection{Análise da Variação da Superfície}

Por fim, nosso próximo objetivo é verificar se a superfície é constante no tempo, o que confirmaria a regra de sticky-moneyness segundo a qual a volatilidade implícita é independente no tempo das variáveis moneyness e maturidade $\left(\sigma_{t}^{\mathrm{imp}}(\mathrm{m}, \tau)=\mathrm{cte}\right)$.

Com a intenção de verificar se a verificar se a superfície de volatilidade pode ser considerada constante no tempo, analisaremos qual o desvio padrão da superfície de volatilidade e se esta variação média diária é significativa a ponto de alterar o preço de uma opção.

O desvio padrão dos M pontos da superfície são calculados do seguinte modo:

$$
\begin{aligned}
& \operatorname{desvpad}\left\{\hat{\sigma}^{\mathrm{imp}}\left(\mathrm{m}_{\mathrm{i}}, \tau_{\mathrm{j}}\right)\right\}=\sqrt{\frac{1}{\mathrm{~T}-1} \cdot \sum_{\mathrm{t}=1}^{\mathrm{T}}\left[\hat{\sigma}_{\mathrm{t}}^{\mathrm{imp}}\left(\mathrm{m}_{\mathrm{i}}, \tau_{\mathrm{j}}\right)-\bar{\sigma}^{\mathrm{imp}}\left(\mathrm{m}_{\mathrm{i}}, \tau_{\mathrm{j}}\right)\right]^{2}} \\
& \quad \mathrm{i}=1, \ldots, \mathrm{Lm} \mathrm{e} \mathrm{j}=1, \ldots, \mathrm{L} \tau
\end{aligned}
$$

Com:

$$
\bar{\sigma}^{\mathrm{imp}}\left(\mathrm{m}_{\mathrm{i}}, \tau_{\mathrm{j}}\right)=\frac{1}{\mathrm{~T}} \cdot \sum_{\mathrm{t}=1}^{\mathrm{T}} \hat{\sigma}_{\mathrm{t}}^{\mathrm{imp}}\left(\mathrm{m}_{\mathrm{i}}, \tau_{\mathrm{j}}\right)
$$

De modo a analisar se os valores de desvio padrão obtidos são significativos, veremos qual o impacto no preço de uma opção que esta variação de volatilidade traria. Para isso, olharemos a variável $v$ (vega), que representa a sensibilidade dos preços à volatilidade. Para a fórmula de apreçameto de Black-76, que estamos utilizando:

$$
\begin{aligned}
& v \equiv \partial \mathrm{C} / \partial \sigma=\mathrm{F}_{\mathrm{t}} \cdot \mathrm{e}^{-\mathrm{r \tau}} \cdot \varphi\left(\mathrm{d}_{1}\right) \cdot \sqrt{\tau} \\
& \mathrm{d}_{1}=\frac{\ln \left(\frac{\mathrm{F}_{\mathrm{t}}}{\mathrm{K}}\right)+\left(\frac{\sigma^{2}}{2}\right) \cdot \tau}{\sigma \sqrt{\tau}}
\end{aligned}
$$

Com:

$\mathrm{F}_{\mathrm{t}}=$ preço futuro do ativo objeto 
$\mathrm{K}$ = strike da opção

$\mathrm{T}=$ data de exercício da opção

$\mathrm{t}=$ instante de negociação

$\tau=\mathrm{T}-\mathrm{t}=$ maturidade

$\sigma=$ volatilidade do ativo objetivo

$\mathrm{r}=$ taxa de juros sem risco

$\varphi()=$. função normal cumulativa padrão.

A variação percentual do preço das opções, em relação a seu preço médio, em cada um dos $M(M=L m x ~ L \tau)$ pontos da superfície, dada uma variação de 1 desvio padrão da volatilidade é:

$$
\begin{aligned}
& \Delta_{(\%)} \mathrm{C}\left(\mathrm{m}_{\mathrm{i}}, \tau_{\mathrm{j}}\right)=\frac{\Delta \mathrm{C}\left(\mathrm{m}_{\mathrm{i}}, \tau_{\mathrm{j}}\right)}{\overline{\mathrm{C}}\left(\mathrm{m}_{\mathrm{i}}, \tau_{\mathrm{j}}\right)}=\frac{\frac{\partial \hat{\mathrm{C}}\left(\mathrm{m}_{\mathrm{i}}, \tau_{\mathrm{j}}\right)}{\partial \sigma} \cdot \operatorname{desvpad}\left\{\sigma\left(\mathrm{m}_{\mathrm{i}}, \tau_{\mathrm{j}}\right)\right\}}{\overline{\mathrm{C}}\left(\mathrm{m}_{\mathrm{i}}, \tau_{\mathrm{j}}\right)} \\
& \Rightarrow \Delta_{(\%)} \mathrm{C}\left(\mathrm{m}_{\mathrm{i}}, \tau_{\mathrm{j}}\right)=\frac{v\left(\mathrm{~m}_{\mathrm{i}}, \tau_{\mathrm{j}}\right) \cdot \operatorname{desvpad}\left\{\sigma\left(\mathrm{m}_{\mathrm{i}}, \tau_{\mathrm{j}}\right)\right\}}{\overline{\mathrm{C}}\left(\mathrm{m}_{\mathrm{i}}, \tau_{\mathrm{j}}\right)} \\
& \mathrm{i}=1, \ldots, \mathrm{Lm} \mathrm{e} \mathrm{j}=1, \ldots, \mathrm{L} \tau
\end{aligned}
$$

Com:

$\Delta_{(\%)} \mathrm{C}=$ variação percentual do preço de uma opção dado uma variação de 1 desvio padrão na superfície de volatilidade

$v=\partial \mathrm{C} / \partial \sigma=$ sensibilidade do preço da opção à volatilidade

$\operatorname{desvpad}\{\sigma\}=$ desvio padrão da superfície de volatilidade

$\overline{\mathrm{C}}=$ preço médio da opção

Para calcular os valores de $\overline{\mathrm{C}}$ e $v$, utilizaremos valores médios observados de taxa de juros, e preço futuro de dólar e volatilidade implícita.

Se o valor da variação percentual do preço da opção em um dia, $\Delta_{(\%)}$, for alto, superando $5 \%$, isto indicará que a superfície de volatilidade não pode ser considerada constante no tempo, pois sua variação, dado um desvio padrão, é suficiente para alterar significativamente o preço das opções. 


\section{Capítulo 5}

\section{Dados utilizados e resultados obtidos}

Apresentamos agora a aplicação prática da teoria descrita anteriormente, sobre o mercado brasileiro, mas especificamente a BM\&F, de opções dólar/real.

\subsection{Dados e Parâmetros Utilizados}

Em nosso estudo, analisamos as opções de dólar/real negociadas na BM\&F desde o dia 01/fev/2007 até o dia 06/jan/2009, que representam 485 dias de negócios. Em média, para cada dia, observam-se 23 opções negociadas, embora algumas destas observações fossem descartadas.

Descartamos as opções cujas volatilidades implícitas fossem abaixo de zero, com maturidades menores que 5 dias e com preço abaixo do limite mínimo teórico de uma opção. Após aplicar estes critérios, ficamos com uma média de 16 opções em cada dia.

Assim, calculamos as volatilidades implícitas e procedemos com a interpolação dos dados conforme a metodologia apresentada. Na interpolação, utilizamos a função peso gaussiana com parâmetros $h_{m}=0.001$ e $h_{\tau}=20$ dias. Estes parâmetros são os que, empiricamente, resultaram em interpolações mais adequadas, evitando sobre ajuste ou então uma superfície que não acompanhasse o efeito smile.

As coordenadas onde faremos as interpolações cobrem, equidistantemente, as coordenadas com mais negócios realizados. Tratam-se de maturidades até 180 dias e moneyness ao redor de 1 . Assim, definimos:

- Coordenadas de Moneyness $=\{0,84 ; 0,86 ; 0,88 ; \ldots ; 1,12 ; 1,14 ; 1,16\}, \mathrm{Lm}=17$ 
Capítulo 5 - Dados utilizados e resultados obtidos

- Coordenadas de Maturidade (dias) $=\{15 ; 45 ; 75 ; 105 ; 135\}, \mathrm{L} \tau=5$;

dessa maneira, são gerados um total de $17 x 5=85$ pontos de interpolação $(M=85)$.

Para a metodologia de análise de componentes principais, determinamos o número de vetores de modo a obter um R-quadrado mínimo da representação da variação da superfície de $75 \%$.

Para a realização dos cálculos, bem como para a apresentação dos dados e resultados obtidos, foi utilizado o software Matlab.

\subsection{Dados Obtidos}

Após o cálculo das volatilidades implícitas e de sua interpolação com os parâmetros descritos anteriormente, temos, como exemplo, os seguintes resultados intermediários (aqui também mostramos as volatilidades implícitas realmente observadas no dia).

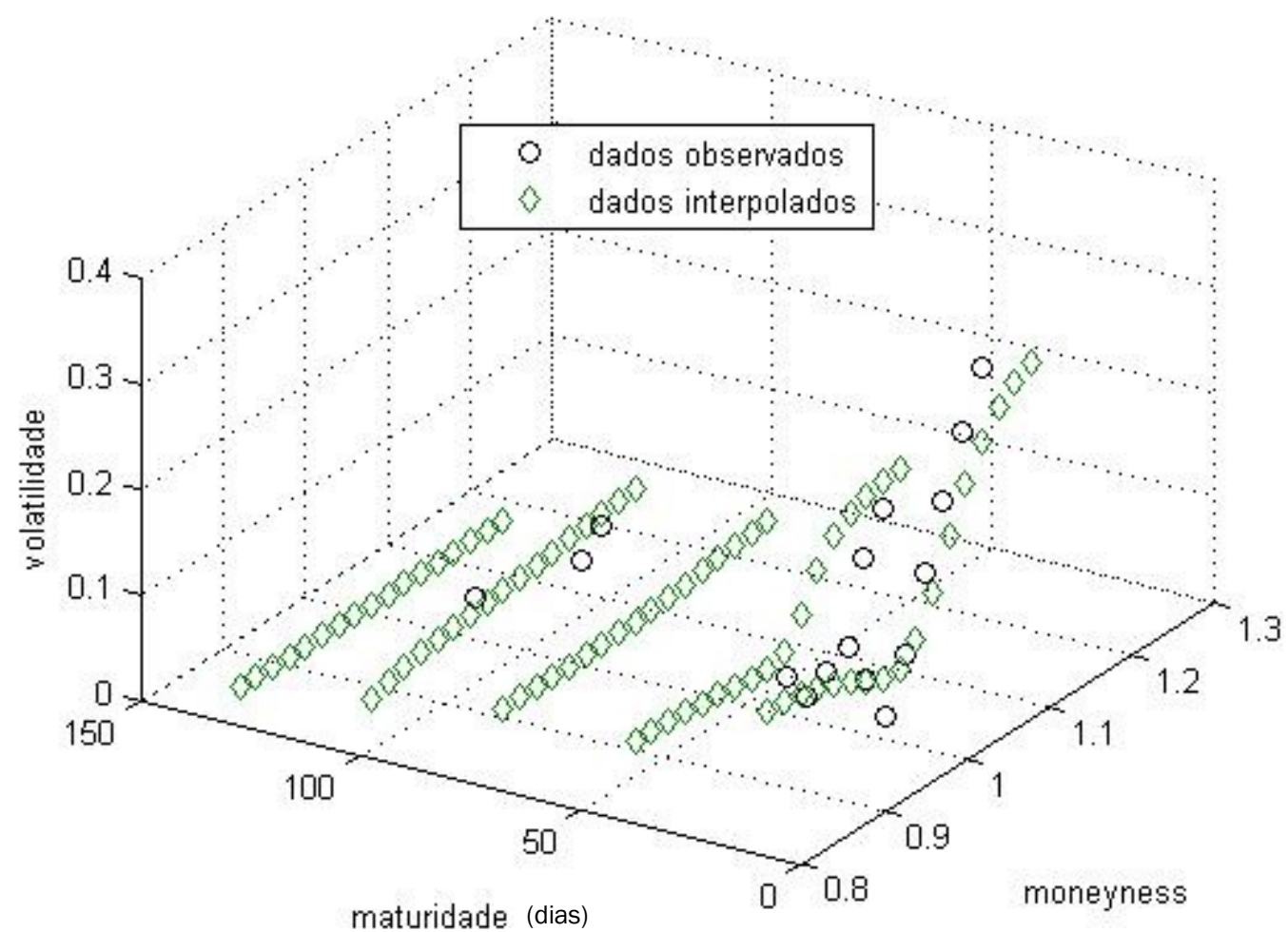

Gráfico 1: Volatilidades implícitas em 08/jan/2007 


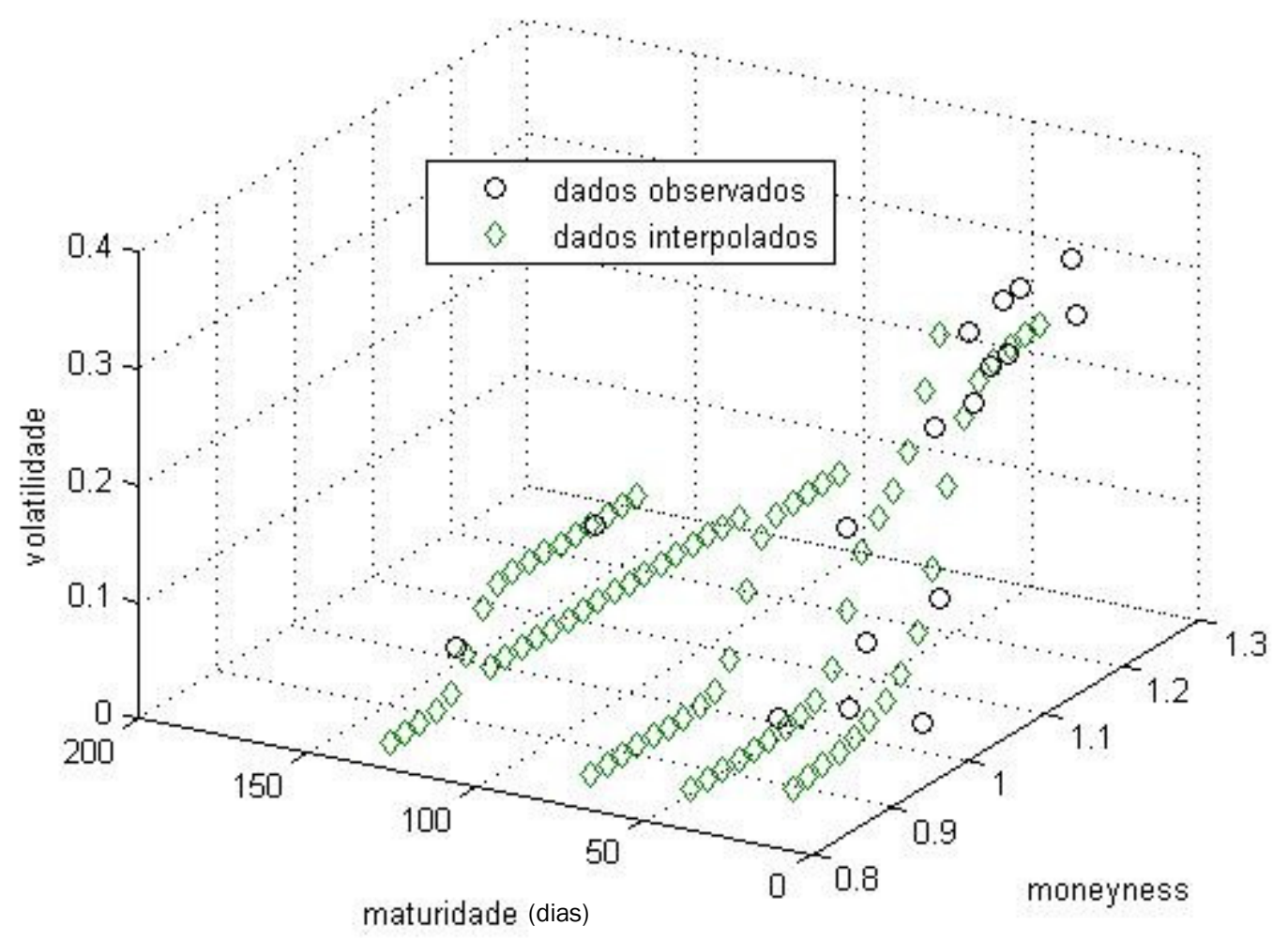

Gráfico 2: Volatilidades implícitas em 14/nov/2008

O próximo passo é a análise de componentes principais que aplicada aos dados retornou que a utilização dos cinco primeiros autovetores como base para representar o processo de variação da superfície de volatilidade eram suficientes para chegar a um R-quadrado de $75 \%$ da variância original. Assim, reduzimos o processo de 85 dimensões, ou 85 pontos discretos, para apenas $5(\mathrm{~N}=5)$. O R-quadrado acumulado de acordo com o número de autovetores utilizados (mostrados apenas para os primeiros 15 autovetores) é: 
Capítulo 5 - Dados utilizados e resultados obtidos

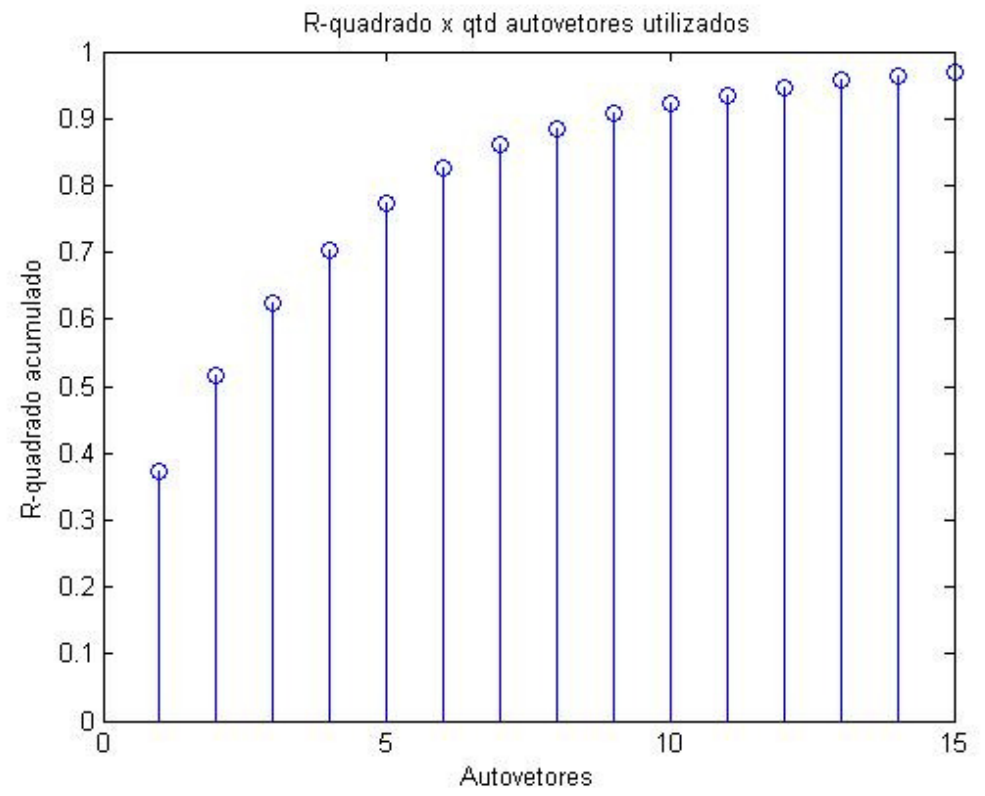

Gráfico 3: R-quadrado da aproximação das variações da superfície de volatilidade

Mostramos a seguir estes cinco autovetores obtidos, de dimensão 85 (85 pontos de superfície de volatilidade), representando-os nas suas coordenadas de moneyness e maturidade, respectivamente $\{0,84 ; 0,86 ; 0,88 ; \ldots ; 1,12 ; 1,14 ; 1,16\}$ e $\{15 ; 45 ; 75 ; 105$; 135\}:

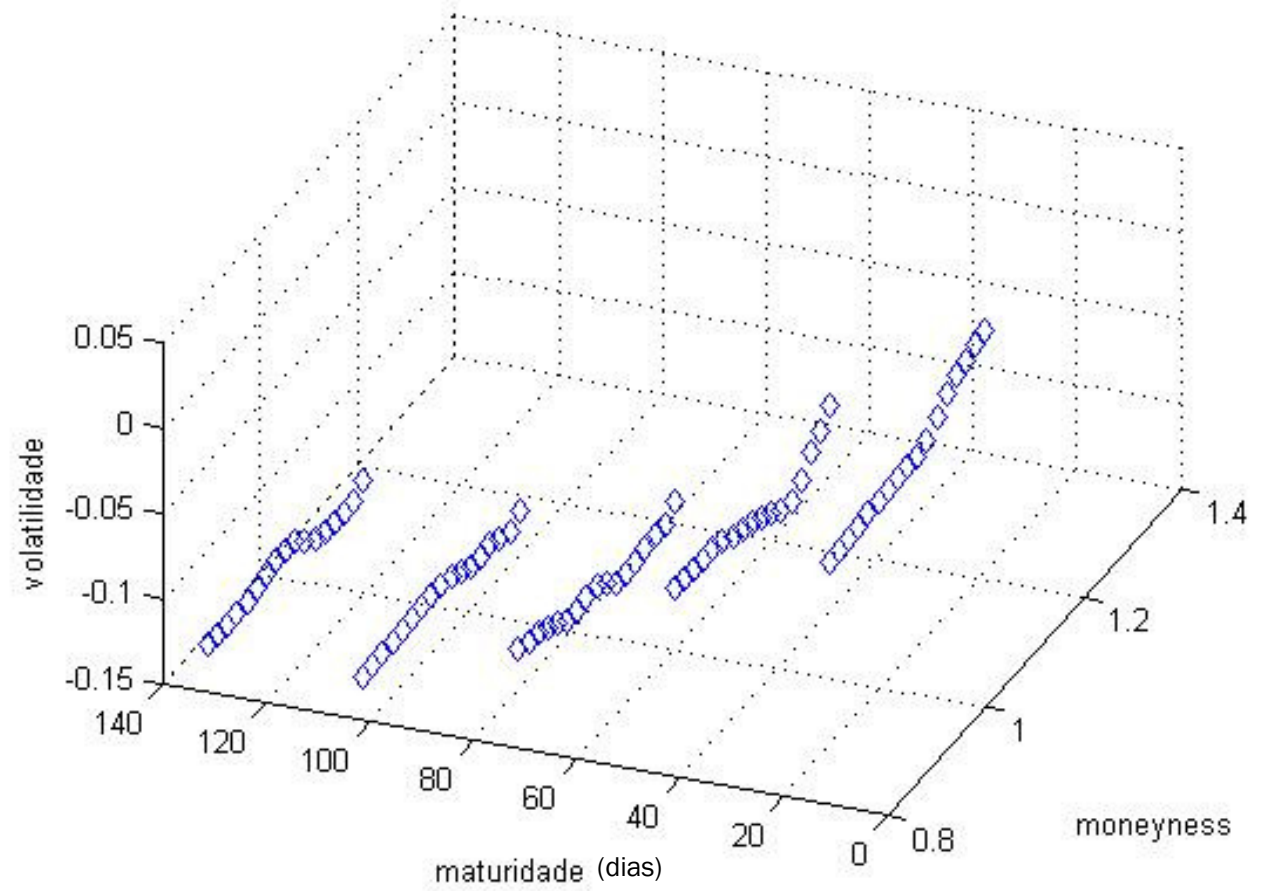

Gráfico 4: $1^{\circ}$ autovetor 
Capítulo 5 - Dados utilizados e resultados obtidos

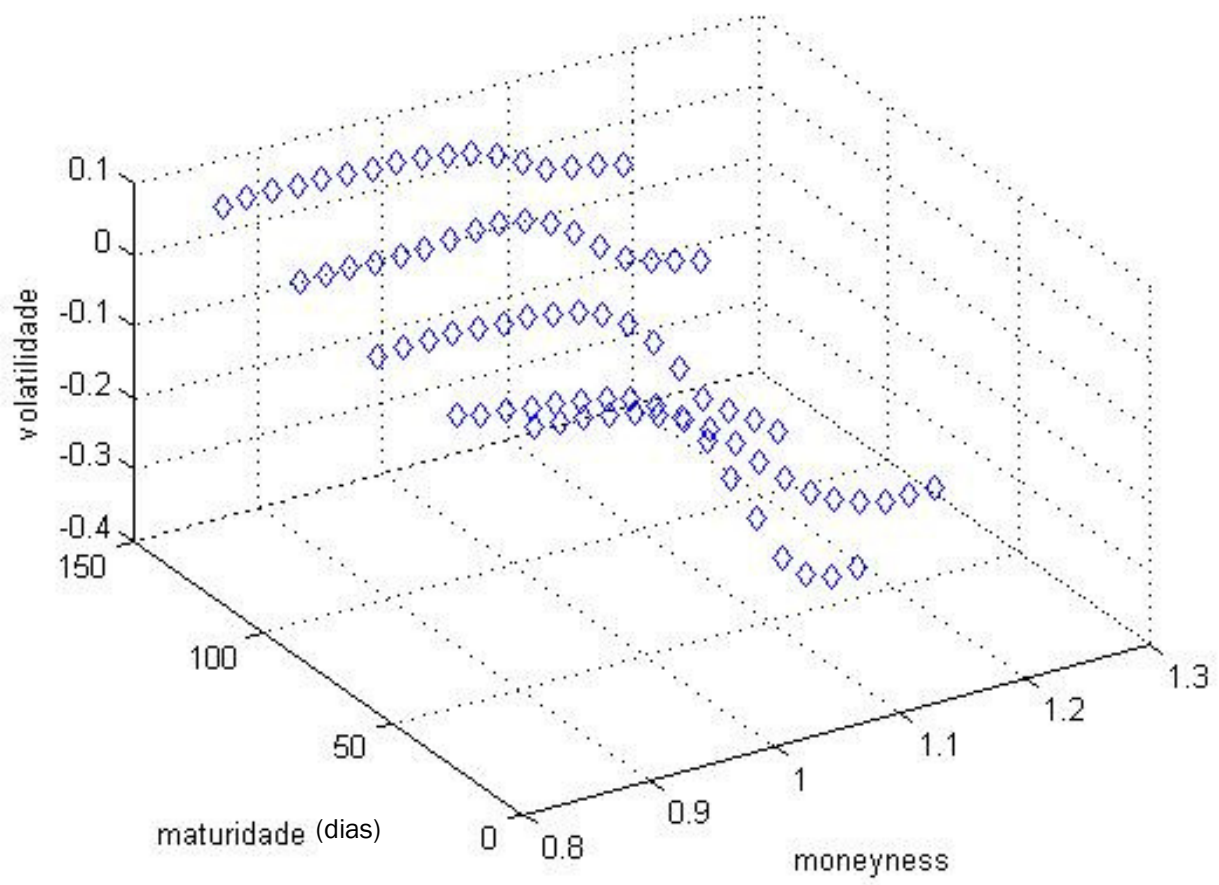

Gráfico 5: $2^{\circ}$ autovetor

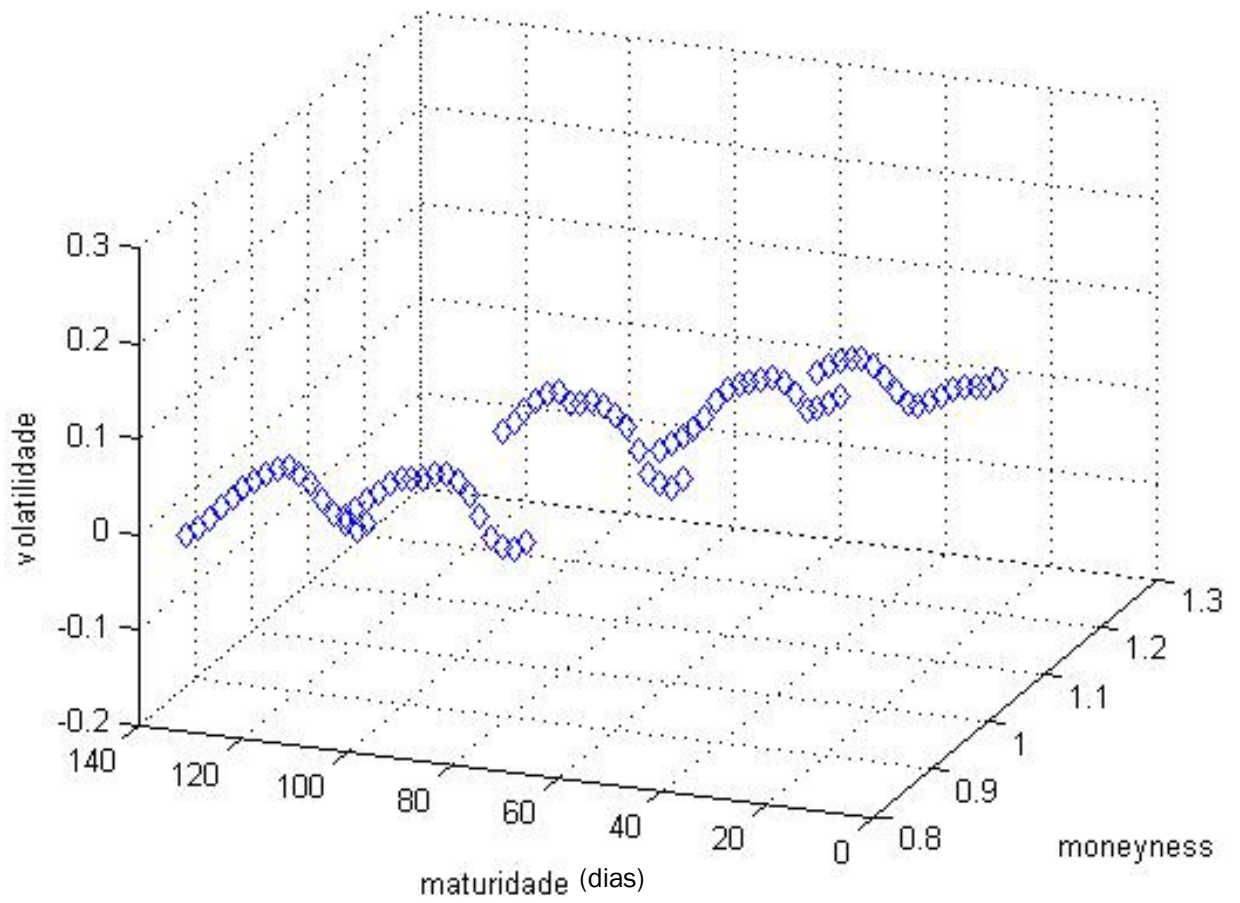

Gráfico 6: $3^{\circ}$ autovetor 
Capítulo 5 - Dados utilizados e resultados obtidos

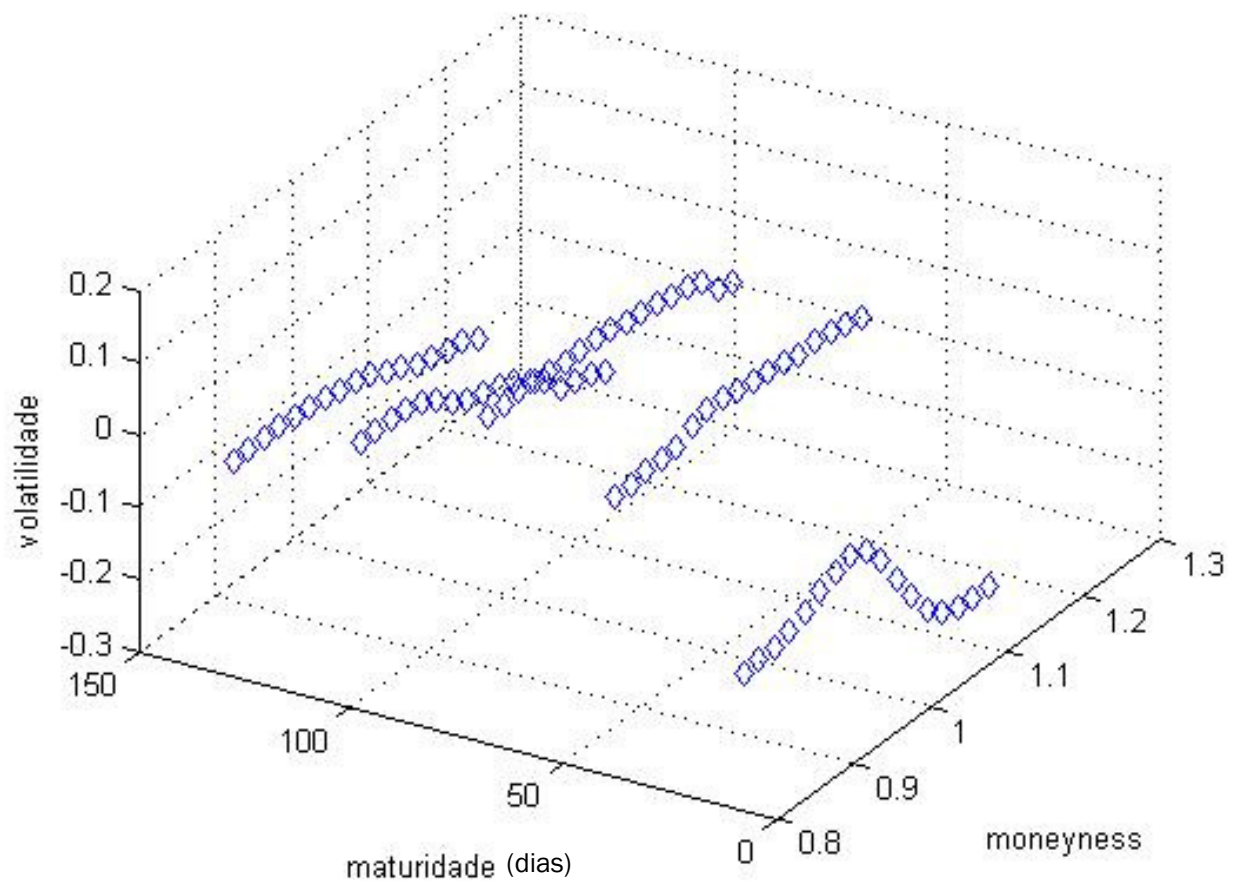

Gráfico 7: $4^{\circ}$ autovetor

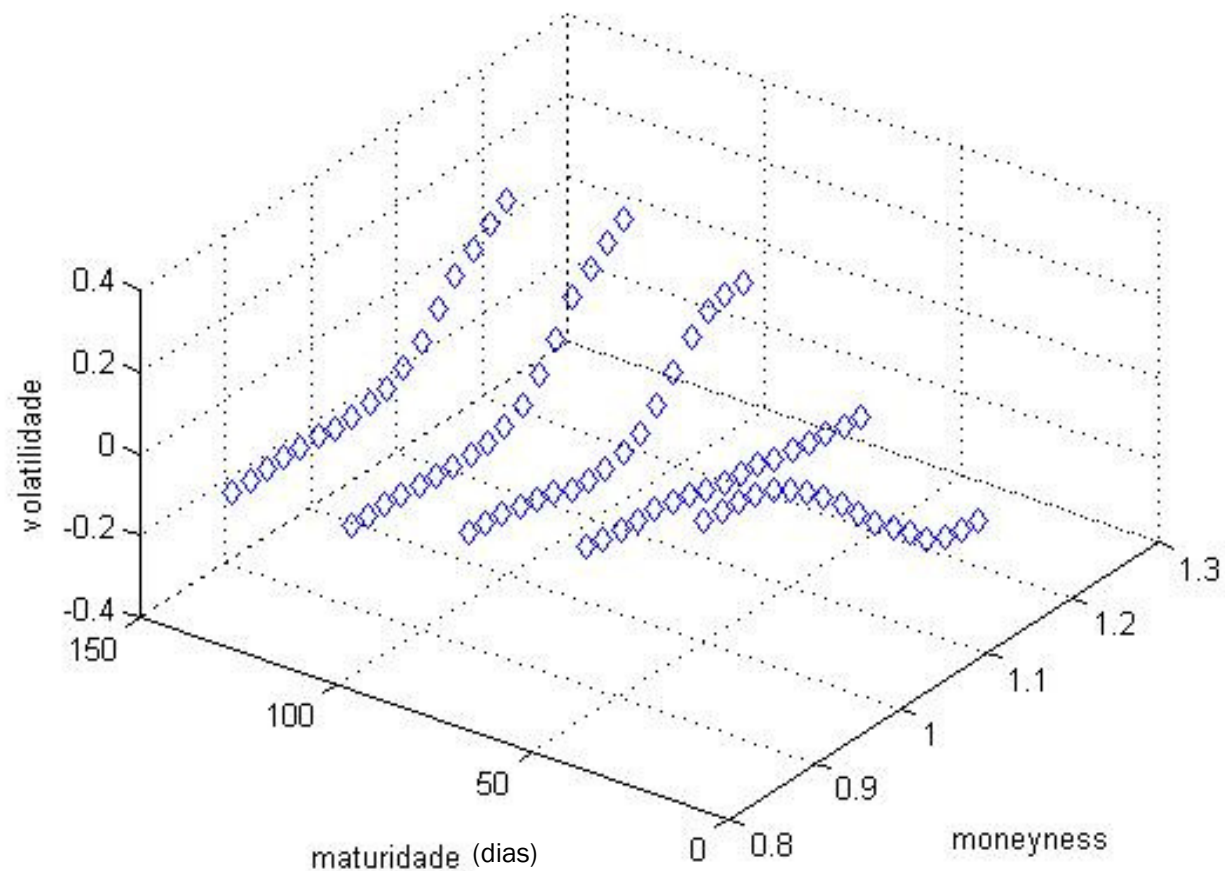

Gráfico 8: $5^{\circ}$ autovetor 
Capítulo 5 - Dados utilizados e resultados obtidos

É possível fazermos algumas observações empíricas de interesse prático sobre os autovetores obtidos. $\mathrm{O} 1^{\mathrm{o}}$ autovetor representa a variação da superfície de acordo com a maturidade, com as volatilidades crescendo conforme as maturidades se reduzem, não havendo variação significativa da volatilidade de acordo com a variação do nível de moneyness.

$\mathrm{O} 2^{\circ}$ autovetor representa o movimento de variação da superfície de acordo com a coordedanada de moneyness: as volatilidades decrescem conforme aumenta-se o moneyness. Este efeito é mais acentuado para as maturidades de 45 e 75 dias.

Já o $3^{\circ}$ autovetor reflete volatilidades crescentes com reduções tanto da maturidade quanto do nível de moneyness. $\mathrm{O} 4^{\circ}$ autovetor mostra valores maiores de volatilidade para maturidades de 45 e 75 dias e menores para as demais maturidades interpoladas: 135,105 e principalmente 15 dias.

Por fim, o $5^{\circ}$ e último autovetor utilizado mostra a inversão do efeito smile, com a volatilidade reduzida para opções com moneyness maiores que $1 \mathrm{em}$ maturidades baixas e crescentes para este mesmo nível de moneyness mas maturidades maiores que 70 dias.

A realização dos parâmetros $Z_{i}$, projeções de $B$ (variação centrada da superfície de volatilidade) nos autovetores $\mathrm{v}_{\mathrm{i}}$, que na metodologia representamos por $\zeta_{\mathrm{i}}$, são mostrados a seguir: 
Capítulo 5 - Dados utilizados e resultados obtidos
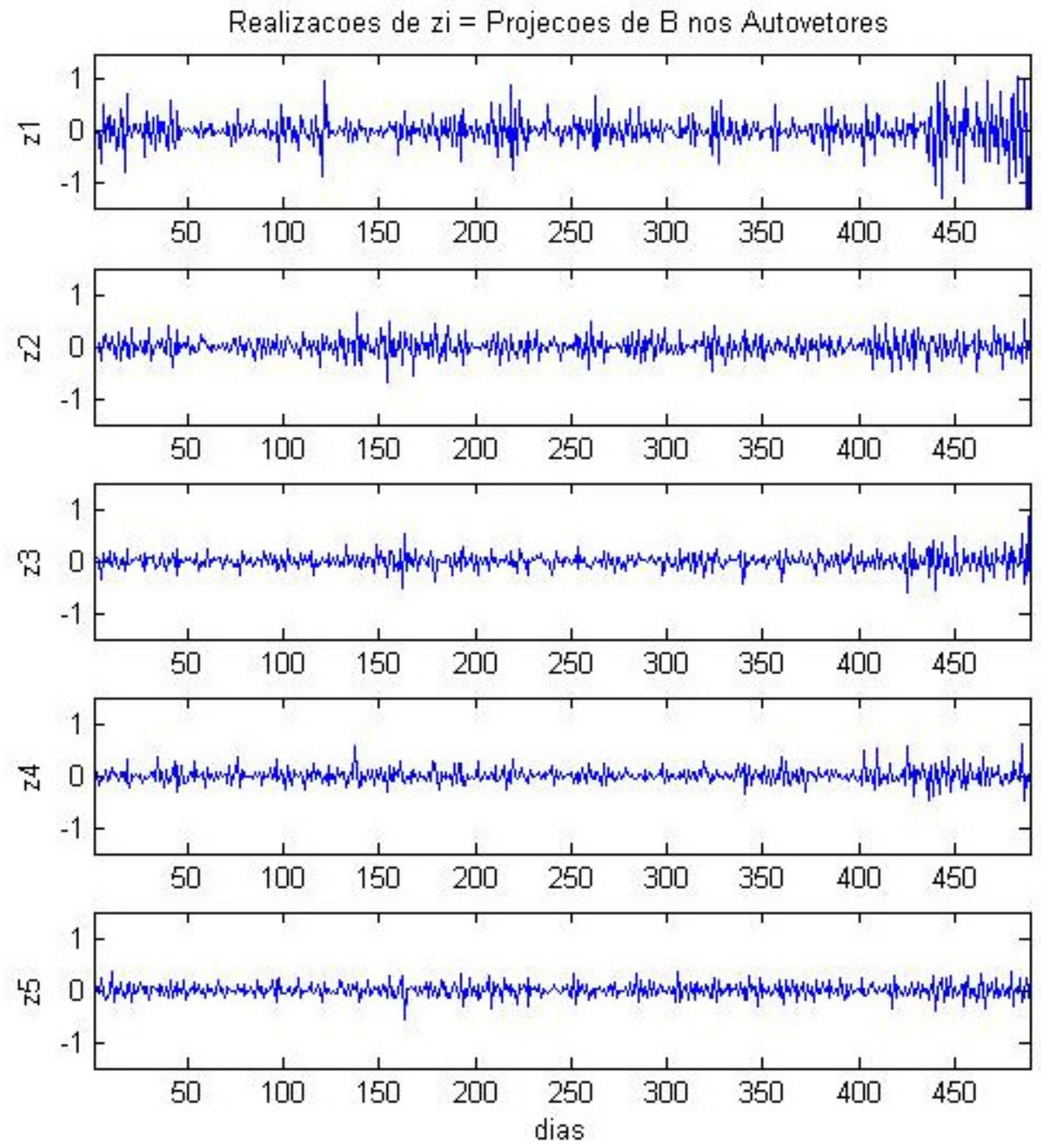

Gráfico 9: Realizações de $z_{i}$

Interessante notar que a realização de $z_{1}$, projeção da variação da superfícies de volatilidade centrada sobre seu principal autovetor, tem sua volatilidade acentuada a partir do $400^{\circ}$ dia de observação, correspondente ao dia 14/08/2008, aproximadamente 1 mês antes da crise cambial (valorização do dólar) iniciada em 2008.

Obtivemos a seguinte matriz de covariância das realizações de $\mathrm{z}_{\mathrm{i}}$ :

$$
\begin{array}{rrrrr}
0,0933 & -0,0000 & -0,0000 & 0,0000 & -0,0000 \\
-0,0000 & 0,0360 & 0,0000 & -0,0000 & 0,0000 \\
-0,0000 & 0,0000 & 0,0271 & -0,0000 & -0,0000 \\
0,0000 & -0,0000 & -0,0000 & 0,0200 & -0,0000 \\
-0,0000 & 0,0000 & -0,0000 & -0,0000 & 0,0172
\end{array}
$$


Capítulo 5 - Dados utilizados e resultados obtidos

Conforme esperado de acordo com a metodologia de análise de componentes principais, os parâmetros apresentam variância decrescente e não são correlacionados.

\subsection{Resultados}

Estimamos o índice de covariância normalizado entre as variações da superfície de volatilidade implícita das opções de dólar e as variações do valor do dólar spot, utilizando a fórmula (4.13), já apresentada no capítulo 4:

$$
\frac{\left\|\operatorname{cov}\left(\tilde{\mathrm{B}}(\mathrm{N}), \Delta \mathrm{S}_{\mathrm{c}}\right)\right\|}{\|\sqrt{\operatorname{var}(\tilde{\mathrm{B}}(\mathrm{N})) \|}\| \cdot \| \sqrt{\operatorname{var}\left(\Delta \mathrm{S}_{\mathrm{c}}\right) \|}}=\frac{\sum_{\mathrm{i}=1}^{\mathrm{N}}\left|\mathrm{E}\left(\mathrm{z}_{\mathrm{i}} \cdot \Delta \mathrm{S}_{\mathrm{c}}\right)\right|}{\sqrt{\sum_{\mathrm{i}=1}^{\mathrm{N}} \lambda_{\mathrm{i}}} \cdot \sqrt{\operatorname{var}\left(\Delta \mathrm{S}_{\mathrm{c}}\right)}}
$$

Para este índice, obtivemos o valor de 0,3358. Se o índice estivesse próximo à 1 , seu valor máximo, concluiríamos que há grande correlação linear entre as variações da superfície de volatilidade e as variações do ativo objeto. Um índice próximo à zero nos levaria a concluir que as variáveis são descorrelacionadas. O valor obtido indica que há uma correlação linear não muito forte entre as variações da superfície de volatilidade de dólar e as variações do ativo objeto (dólar spot).

Outro de nossos objetivos é avaliar se a superfície de volatilidade pode ser considerada constate no tempo. Para isso, verificamos qual o desvio padrão da superfície de volatilidade. O desvio padrão obtido é apresentado no gráfico a seguir: 


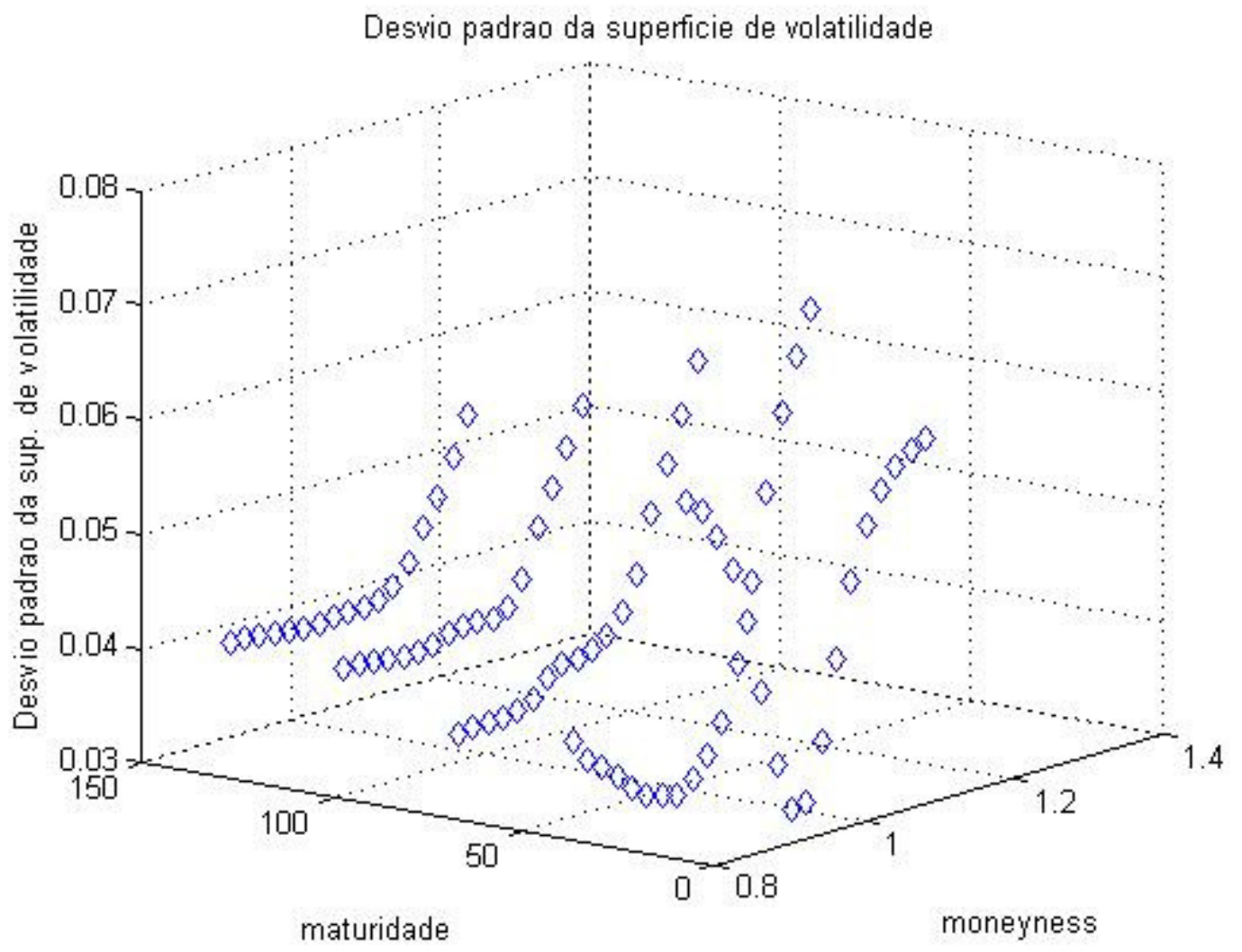

Gráfico 10:Desvio Padrão da Superfície de Volatilidade

Observa-se que a superfície de volatilidade possui desvio padrão entre 3\% e 7\%, sendo maior nas extremidades da dimensão de moneyness.

Estes valores serão considerados representativos se implicarem em uma alteração do preço das opções superior à 5\% em 1 dia. A análise de qual a variação percentual do preço de uma opção dada esta variação de 1 desvio padrão na superfície é feita com a fórmula (4.19) que vamos reproduzir para facilitar o acompanhamento:

$$
\Delta_{(\%)} \mathrm{C}\left(\mathrm{m}_{\mathrm{i}}, \tau_{\mathrm{j}}\right)=\frac{v\left(\mathrm{~m}_{\mathrm{i}}, \tau_{\mathrm{j}}\right) \cdot \operatorname{desvpad}\left\{\sigma\left(\mathrm{m}_{\mathrm{i}}, \tau_{\mathrm{j}}\right)\right\}}{\overline{\mathrm{C}}\left(\mathrm{m}_{\mathrm{i}}, \tau_{\mathrm{j}}\right)}
$$

Onde desvpad $\left\{\sigma\left(\mathrm{m}_{\mathrm{i}}, \tau_{\mathrm{j}}\right)\right\}$ são os valores apresentados no gráfico anterior. Os resultados obtidos para cada ponto da superfície são: 
Capítulo 5 - Dados utilizados e resultados obtidos

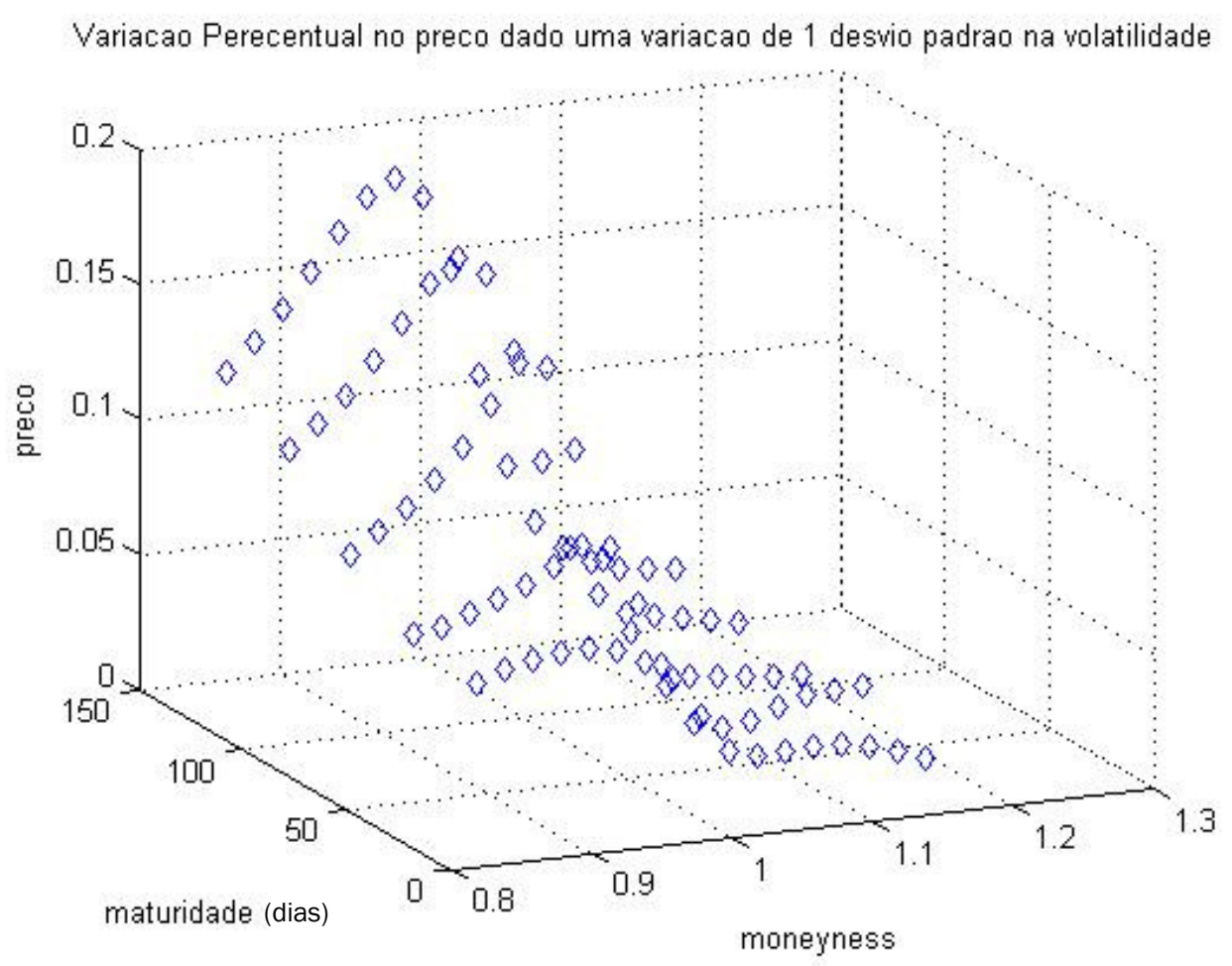

Gráfico 11: Variação percentual teórica do preço das opções

A variação teórica do preço das opções obtida deste modo chega à valores de $18 \%$, sendo a variação média de preço, dos vários pontos estudados na superfície, de 7,3\%. Assim, a variação da superfície de volatilidade implícita altera o preço das opções consideravelmente, ou seja, não é possível considerar a superfície como estática e, portanto, descartamos a regra de sticky-moneyness.

É interessante notar também que pontos com grande desvio padrão da superfície de volatilidade implícita, como os de maturidade 15 dias e moneyness acima de 1, apresentam pequena variação percentual, $\Delta_{(\%)} \mathrm{C}$, em seu preço enquanto pontos de grande maturidade e moneyness próximo a 1 apresentam pequeno desvio padrão da superfície, mas grande variação percentual de seu preço. Isso se deve às diferentes sensibilidades dos preços à volatilidade implícita, que é representada pela variável $v$. 


\section{Capítulo 6}

\section{Conclusão}

Observando as opções européias de dólar negociadas na BM\&F por mais de um ano, pudemos calcular suas volatilidades implícitas e em seguida montar/interpolar uma superfície de volatilidade discreta, que, como esperado pela literatura, não eram constantes nas dimensões de moneyness e maturidade.

O processo de variação diária dos pontos calculados desta superfície de volatilidade foi reproduzido via análise de componentes principais, simplificando sua análise estatística ao reduzir a dimensão do processo em variáveis ortogonais. A partir desta análise, algumas conclusões interessantes, já obtidas em outros estudos, porém em outros mercados, puderam ser alcançadas.

A decomposição da variação da volatilidade implícita em 5 autovetores preservando $75 \%$ da variância original deste processo é um dos resultados importantes de nosso trabalho. A partir dele conclui-se que, em uma carteira com diversas opções cambiais, a volatilidade implícita deve ser representada por 5 fatores de risco diferentes e não por um único fator.

É possível concluir também, analisando os resultados obtidos, que no mercado de opções européias de dólar negociadas na BM\&F as variações diárias de sua superfície de volatilidade possuem uma fraca correlação com as variações do dólar spot, o ativo objeto em questão. A covariância normalizada, índice que pode variar de 0 e 1, entre as componentes que representam a variação da superfície de volatilidade e a variação do valor do dólar spot foi de 0,34. Assim, podemos concluir que a volatilidade implícita destas opções deve ser tratada como um fator de risco à parte, muito embora suas 
variações estejam correlacionadas com a variação do valor do ativo objeto de modo significativo.

Este resultado está em linha com a apresentado em outros mercados, como o índice de ações S\&P500, estudado por Cont e Fonseca (2002) e Skiadopoulos; Hodges e Clewlow (2000) e com o FTSE1000. Uma comparação mais direta dos resultados não é possível, pois as metodologias para apuração desta correlação são diferentes nos 3 trabalhos

Também avaliamos a variância da superfície de volatilidade, de modo a determinar a confiabilidade da regra de sticky-moneyness, segundo a qual a superfície de volatilidade deveria ser constante. Analisamos se esta variância é relevante por meio do seu impacto no preço das opções, utilizando a variável $v$ (vega).

Em nosso trabalho, verificamos que as variações nas volatilidades implícitas das opções de dólar da BM\&F são significativas e alteram em um dia o preço das opções negociadas, em média, 7,3\% e portanto concluímos que a regra de stickymoneyness não é válida para o mercado de opções de dólar da BM\&F. Esta conclusão está em linha ao apresentado em estudos anteriores aplicados a outros mercados. Alexander (2001) e Fengler; Härdle e Mammen (2005) aplicaram seus estudos em mercados de índices de ações, o DAX Alemão e o FTSE100, e também refutaram esta regra.

\subsection{Perspectivas Futuras}

A partir da análise de componentes principais sobre as volatilidades implícitas das opções de dólar que realizamos, abre-se a possibilidade de estudar a criação de estratégias de negociação de opções cambiais. Estas estratégias seriam baseadas em uma representação mais detalhada do processo estocástico do movimento da superfície de volatilidade.

Também sugerimos como continuação de nosso trabalho o estudo de técnicas mais precisas para o gerenciamento de risco de volatilidades. Este estudo se beneficiaria 
Capítulo 6 - Conclusão

da construção, realizada em nosso trabalho, da superfície de volatilidade em variáveis ortogonais, onde cada variável seria considerada como um fator de risco à parte.

Por fim, outra possibilidade de continuação para nossos estudos é o desenvolvimento de uma metodologia de apreçamento de derivativos cambiais exóticos baseada na superfície de volatilidade implícita e suas variações. 


\section{Referência Bibliográfica}

AÏT-SAHALIA, Y.; LO, A. Nonparametric estimation of state-price densities implicit in financial asset prices. J. Finance, v. 53, p.499-548, 2000.

ALEXANDER, C. Principal Component Analysis of Volatility Smiles and Skews. Working paper, ISMA Centre, University of Reading, 2001.

AVELLANEDA, M.;FRIEDMAN, C.; HOLMEs, R. ; SAMPERI, D. Calibrating volatility surfaces via relative-entropy minimization. Applied Mathematical Finance, v. 4, p.37-64, 1997.

BATES, D. Empirical Option Pricing: A Retrospection. Journal of Econometrics, v. 116, p.387-404, 2003

BLACK, F. The pricing of commodity contracts. Journal of Financial Economics, v. 3, p167-179, 1976

BLACK, F.; SCHOLES, M. The pricing of options and corporate liabilities. J.

Political Economy, v. 81, p.637-654, 1973

CARR, P.; MADAN, D. A note on sufficient conditions for no arbitrage. Finance Research Letters, v. 2, p.125-130, 2005

CONT, R.; FONSECA, J.;DURRLEMAN, V. Stochastic models of implied volatility surfaces. Economic Notes, v. 31(2), p.361-377, 2002

CONT, R.; FONSECA, J. Dynamics of implied volatility surfaces. Quantitative Finance, v. 2(1), p.45-60, 2002.

COX, J. C. ; ROSS, S. A. ; RUBINSTEIN, M. Option Pricing: A Simplified Approach. Journal of Financial Economics, v. 7, p229-263, 1979.

DERMAN, E. Regimes of volatility. Risk, v. 12(4), p.55-59, 1999

DERMAN, E.; KANI, I., "The volatility smile and its implied tree". Quantitative Strategies Research Notes, Goldman Sachs,1994

DUMAS, B.; FLEMING, J.; WHALEY, R. E. Implied volatility functions: empirical tests, J. Finance, v. 8, p.2059-2106, 1998

DUPIRE, B. Pricing with a smile, Risk, v. 7. p.18-20, 1993 
FAHRMEIR, L.; KNORR-HELD, L. Dynamic and semiparametric models. Smoothing and Regression: Approaches, Computation and Application (Edited by M. Schimek). Wiley, New York, 2000

FENGLER, M.; HÄRDLE, W.; MAMMEN, E. Implied Volatitlity String Dynamics. SFB 649 Discussion Paper, Hümboldt-Universität, Berlin, 2003

FENGLER, M.; HÄRDLE, W.; MAMMEN, E. A dynamic semiparametric factor model for implied volatility string dynamics. SFB 649 Discussion Paper 020, Humboldt-Universität, Berlin, 2005.

FENGLER, M.; WANG, Q. Fitting the smile revisited: A least squares kernel estimator for the implied volatility surface. SFB 373 Discussion Paper 25, Humboldt-Universität, Berlin, 2003

FOUQUE, J.-P.; G. PAPANICOLAOU, G.; SIRCAR, R.; SOLNA, K. Timing The Smile. Wilmott Magzine, March 2004

GUIMARÃES, B. V.; SILVA, M. E. A Possibilidade de Saltos no Câmbio Implícita nos Prêmios das Opções. Revista Brasileira de Economia, v. 56(3), p.397 - 428, 2002

HE, X.; NG, P. COBS: Qualitatively Constrained Smoothing via Linear Programming. Discussion Paper, University of Illinois, 1999

JACKWERTH, J.C. Option-Implied Risk-Neutral Distributions and Implied Binomial Trees: A Literature Review, Journal of Derivatives, v. 6, p.1-17, 1999

LAURINI, M. Imposing No-Arbitrage Conditions In Implied Volatility Surfaces Using Constrained Smoothing Splines. IBMEC Working Paper, WPE 41, 2007

LEON, C.; OKER, B. Analyzing the dynamics behind the volatility surface: The Euro Swap Rate Case. 42p. Dissertação (Master of Science in Banking and Finance) - École des HEC. University of Lausanne, 2005

MERTON, R.C. Theory of rational option pricing. Bell Journal of Economics and Management Science, v. 4, p.141-183, 1973

MEUCCI, A. Risk and Asset Allocation. Springer, 2005.

RUBINSTEIN, M. Implied binomial trees, J. Finance, v. 49, p.771-818, 1994

SKIADOPOULOS, G.; HODGES, S.; CLELOW, L. Dynamics of the S\&P500 implied volatility surface, Rev. Derivatives Res. , v. 3, p.263-82, 2000 